\title{
Kinetic mixing and portal matter phenomenology
}

\author{
Thomas G. Rizzo* \\ SLAC National Accelerator Laboratory, 2575 Sand Hill Road, Menlo Park, California 94025, USA
}

(Received 1 February 2019; published 18 June 2019)

\begin{abstract}
Dark photons are interesting as potential mediators between the dark matter sector and the fields of the Standard Model (SM). The interaction of the dark photon, described by a broken $U(1)_{D}$ gauge symmetry, with the SM is usually generated at the one-loop level via kinetic mixing through the existence of portal matter, here assumed to be fermionic, which carries both a dark charge as well as a SM $U(1)_{Y}$ hypercharge. For theoretical consistency, as well as for many phenomenological reasons, this portal matter must be vectorlike with respect to the SM and dark gauge groups and, in particular, is shown to be allowed only to transform as vectorlike copies of the usual SM fields. The dark Higgs that is responsible for the breaking of $U(1)_{D}$ can then generate a mixing between the portal matter and SM fields with the same electric charge thus altering the dark photon/portal matter interactions with (at least some of) the SM fields and also providing a path for the portal matter fields to decay. In this paper we briefly explore the phenomenology of some specific simple models of this portal matter including, for the case where the portal matter is leptonic in nature, their potential impact on experiments probing low energy parity violation and the g- 2 of the muon. In the case of color triplet, bottom quarklike portal matter, their direct pair and single production at the LHC is shown to be observable in final states that include missing $E_{T}$ and/or very highly boosted lepton jets together with pairs of high- $p_{T} b$-jets that can be used to trigger on such events. These signatures are quite distinct from those usually employed in the search for vectorlike quarks at the LHC and, furthermore, we demonstrate that the conventional signal channels for vectorlike quarks involving the SM Higgs and gauge fields are essentially closed in the case of portal matter. Many other more complex, and more realistic, portal matter scenarios of the type discussed here are possible which can lead to wide-ranging signatures in various classes of experiments.
\end{abstract}

DOI: 10.1103/PhysRevD.99.115024

\section{INTRODUCTION}

The nature of dark matter (DM) remains as one of the great mysteries facing particle physics. For DM to achieve its observed abundance, it is probable that it must have some new, nongravitational interaction(s) with the fields of the Standard Model (SM) - but such interactions are likely to be far weaker than the usual ones with which we are familiar. Traditionally, both axions [1,2] and WIMPs [3] have been the leading candidates for DM, originating in top-down frameworks that addressed other issues such as the strong $C P$ problem and naturalness. Searches for such particles have so far obtained only null results and while these important experiments are still evolving the set of possible DM candidates continues to grow as does the possible ways to look for them [4,5]. Among these, one of

\footnotetext{
rizzo@slac.stanford.edu
}

Published by the American Physical Society under the terms of the Creative Commons Attribution 4.0 International license. Further distribution of this work must maintain attribution to the author(s) and the published article's title, journal citation, and DOI. Funded by SCOAP . the most interesting and directly testable possibilities that has garnered significant recent attention is the kinetic mixing/vector portal model [6,7]. In its most simple form, this setup assumes the existence of a dark photon, i.e., the gauge field of a new $U(1)_{D}$, that is spontaneously broken through the vacuum expectation value (vev) of a complex SM singlet dark Higgs field, $\langle S\rangle=v_{s} / \sqrt{2}$, which here we will assume to lie in the range $v_{s} \sim 0.1$ to a few $\mathrm{GeV}$. The DM field then carries a nonzero dark charge, $Q_{D}$, so that it can couple directly to the dark photon. On the other hand, the SM fields do not carry any dark charges under this new $U(1)_{D}$ so that the interactions of the dark photon with the SM fields are loop induced via the kinetic mixing (KM) of the dark photon with the usual SM $U(1)_{Y}$ hypercharge field, $B_{\mu}$, whose mixing strength is characterized by a parameter, $\epsilon \sim 10^{-(3-5)}$ for dark photons of mass $\sim 0.1-1 \mathrm{GeV}$ or so. The vacuum polarizationlike diagram that generates this interaction is the result of the existence of an important (though often neglected) new set of fields which carry both SM as well as dark charges; we will refer to such dual-charged states as portal matter in what follows. This portal matter thus plays an essential role in all models where the DM interactions with the SM are 
induced by KM. As will be further discussed below, assuming that the $\mathrm{KM}$ is indeed generated only at oneloop to have a phenomenologically interesting strength, in order to obtain a nonzero and yet finite result (as might be expected in UV-complete scenarios) several, nondegenerate portal matter fields are required, the product of whose charges must satisfy the condition $\sum_{i} Q_{Y_{i}} Q_{D_{i}}=0$. What are these portal matter states and how do they impact nonDM phenomenology? These portal matter fields which form a necessary ingredient of this setup have so far received very little attention much less detailed study in the literature [8] particularly in the case where they carry nontrivial SM $S U(3)_{c}$ charges. To rectify this situation, it is the nature of these portal matter fields, their interactions with the dark photon, as well as with the familiar SM particles that will be of interest to us below. We will see that even after the obligatory constraints are satisfied there remains considerable flexibility in the identity and spectrum of the portal matter states which can result in broad phenomenological implications which we can only begin to examine here. How these portal matter states may fit into a more complete theoretical structure will be discussed elsewhere.

If the portal matter fields are fermionic, which we will argue below is the most likely scenario and which we will assume in the following discussion, they must be vectorlike with respect to both the $U(1)_{D}$ and the SM gauge groups in order to avoid various gauge anomalies, precision electroweak constraints and the additional constraints arising from the Higgs boson's production and decay properties as measured at the LHC. Such vectorlike fermions (VLF) are then qualitatively similar in nature to those that have been well studied in various extensions of the SM [9] and which have been sought at the LHC without success (so far) by both the ATLAS and CMS Collaborations [10]. The difference in the present case is that these portal matter fields also carry dark charges so that they cannot mix with the correspondingly charged SM fields via a tree-level Yukawa coupling with the SM Higgs. This implies that their decays to final states involving either the SM Higgs or the SM gauge bosons are highly suppressed yet these are the very modes being searched for at the LHC. However, such mixings between the portal matter and the fermion fields of the SM can occur when the $U(1)_{D}$ charges of these portal matter fields allow for an analogous Yukawa coupling to the dark Higgs which, as we will see below, leads to quite a different phenomenology and production signatures at the LHC now involving long-lived states arising from the dark photon and dark Higgs. Furthermore, we will show that such mixings are required in order to satisfy numerous lifetime constraints on such new particles carrying SM charges. As we will discuss, the existence of such states can also have direct impact on other experiments performed at lower energies depending upon their specific SM quantum numbers. Much of this phenomenology, as we will see, has been previously encountered in dark photon models in other contexts at the qualitative level. However, its specific nature and origin in the present analysis, as we will see below, are quite different quantitatively from that most frequently encountered in the literature.

The outline of this paper is as follows: Section II begins by providing a general overview of the $\mathrm{KM} /$ dark photon setup and the role that portal matter plays within it. After specializing to the case of the portal matter being a set of SM-like vectorlike fermions, the mixing of these portal matter fields with those of the SM are analyzed in detail in the most minimal scenario involving only a single SM flavor pair of portal matter fields. As noted above, much of this phenomenology will be familiar at the qualitative level but will be shown to generally differ quantitatively from these other more well-known results. Section III provides a noncomprehensive survey of some of the possible phenomenological implications of the existence of the mixing between the SM fields and the portal matter but which depend on the detailed SM quantum numbers assumed for the portal matter states. The modifications of the dark photon couplings to the SM fields that are induced by this mixing are then considered; it is pointed out that these, generally parity-violating, contributions can be comparable in strength to those induced by the familiar KM. The portal matter/SM mixing also induces off-diagonal couplings of these portal matter/SM fields to both the dark photon and the dark Higgs. In the case of color-singlet portal matter, if the portal matter transforms like a SM right-handed electron then such new parity-violating effects may be observable in low energy experiments such as MOLLER. If the portal matter is $\mu_{R}$-like in nature, this leads to multiple new contributions to the g- 2 of the muon, whose experimental value is known to differ from the SM prediction by $\sim 4 \sigma$. We note that if the portal matter were similar to the left-handed lepton doublet, then the mixing with the vectorlike fermion $N$ induces a new low- $Q^{2}$ interaction between neutrinos and quarks which may impact measurements at, e.g., MiniBooNE or DUNE. Lastly, and in more detail, we consider the setup where the portal matter transforms as a color triplet, in particular like $b_{R}$ in the $\mathrm{SM}$; this is the main emphasis of the current work. In such a case a variety of possible signals can arise from their pair production at the LHC. In addition to the two, high- $p_{T}$, $b$-jets produced in portal matter pair decay which can be employed as a tag, the final state is shown to include missing $E_{T}$, producing a supersymmetry (SUSY)-like signature, and/or displaced lepton jets which are atypical due their very large boosts. The case where the $b_{R}$-like portal matter is singly produced is also briefly discussed, is shown to lead to asymmetrically boosted lepton jets and to potentially have a significant production rate at the LHC. A comparison to the case where the portal matter transforms as $t_{R}$-like is also briefly made. Section IV contains a final discussion and our conclusions. 
An appendix summarizes the most important couplings appearing in the present analysis.

\section{PORTAL MATTER PHENOMENOLOGY SETUP}

In this paper we will be particularly interested in the range of dark matter and dark photon masses $\sim 0.1-1 \mathrm{GeV}$ and below that will be accessible in, e.g., future fixed target and light DM direct search experiments. Within this context, consider a set of vectorlike fermions, $F_{i}$, with masses $m_{i}$ and whose hypercharges and corresponding dark charges are denoted by $Q_{Y_{i}}$ and $Q_{D_{i}}$, respectively. With KM absent at tree level, then, in conventional normalization [7], the familiar 1-loop vacuum polarizationlike graph coupling the two corresponding gauge fields will lead to experimentally interesting values $[4,5]$ for the $\mathrm{KM}$ parameter $\epsilon \sim 10^{-(3-5)}$ given by $\left(c_{W}=\cos \theta_{W}\right)[11]$

$$
\epsilon=c_{W} \frac{g_{D} g_{Y}}{12 \pi^{2}} \sum_{i} Q_{Y_{i}} Q_{D_{i}} \ln \frac{m_{i}^{2}}{\mu^{2}}
$$

where obtaining a finite result (as might be expected in UV-complete scenarios) requires that these fields be nondegenerate and satisfy the constraint $\sum_{i} Q_{Y_{i}} Q_{D_{i}}=0$; here $g_{Y, D}$ are the hypercharge and dark gauge coupling constants, respectively. We will assume that $g_{D} \sim 0.1-0.3$ in the numerical analysis that follows. The very simplest possibility, which we will consider here, is thus where there are only two such vectorlike fermions, $F_{1,2}$, with different masses, having the same value of the SM hypercharge but with simultaneously opposite values for $Q_{D}{ }^{1}$; we will take this numerical value to be unity, $\left|Q_{D_{i}}\right|=1$, without loss of generality in the present analysis. Thus, in this limited discussion, we will constrain ourselves to the case where the portal matter consists of a single pair of fields that have identical SM characteristics. Clearly, we can generalize far beyond this simplifying assumption to more complex, but perhaps more realistic and interesting, situations but we will leave this to later work. Given these considerations, we then obtain numerically

$$
\epsilon \simeq 1.0\left(\frac{g_{D}}{0.1}\right) N_{c} Q_{Y} \frac{\ln \left(m_{2} / m_{1}\right)}{\ln 1.5} \times 10^{-4}
$$

a value which is consistent with our expectations $[4,5]$ and where $N_{c}$ is the number of colors of the portal matter fields. Since these portal matter states carry both SM and dark charges they must also be unstable to conform with cosmological constraints and so a decay path for them

\footnotetext{
${ }^{1}$ The opposite charge assignment possibility provides some interesting model building challenges if the portal matter transforms in a nontrivial manner under the $\mathrm{SM} S U(2)_{W}$ or $S U(3)_{c}$ gauge groups.
}

must be generated in any realistic theory. In practice this implies that they must mix with the analogous SM fields as we will discuss further below.

It should again be stressed that this rather simple scenario need not be the one that is actually realized in nature and many interesting combinations of the vectorlike fermion fields playing the role of portal matter are possible. For example, one can imagine that in more UV-complete scenarios, e.g., portal matter contributions from color triplet, weak isosinglets naturally "canceling" against those from color singlet, weak isodoublets as in a $5+\overline{5} \mathrm{SU}(5)$ like representation as happens in the case of KM in GUTS. Such possibilities may open a window to a more realistic UV completion of the present toy model than we contemplate here [12]. Indeed, such possibilities will be considered elsewhere but one should be mindful of their potential phenomenological impact which may lie simultaneously in multiple phenomenological sectors and in having broad experimental implications. We note in passing that if we had instead chosen the portal matter to be complex scalars without vevs, it is much more difficult to arrange for them to decay via mixing with the analogous SM field, i.e., the Higgs, which is the only available SM scalar, without further augmentation of the model.

Once a value of $\epsilon \neq 0$ is obtained this leads to the now standard development $[6,7]$ (which we now briefly review to set the stage for later discussion) via the Lagrangian

$$
\mathcal{L}=\mathcal{L}_{1}+\mathcal{L}_{2}+\mathcal{L}_{H S}+\mathcal{L}_{\text {Portal }}
$$

with the $\mathcal{L}_{1}$ piece of the action describing the dark photon field, $\hat{V}$, the corresponding hypercharge gauge field, $\hat{B}$, and the rest of the SM:

$\mathcal{L}_{1}=-\frac{1}{4} \hat{V}_{\mu \nu} \hat{V}^{\mu \nu}-\frac{1}{4} \hat{B}_{\mu \nu} \hat{B}^{\mu \nu}+\frac{\epsilon}{2 c_{w}} \hat{V}_{\mu \nu} \hat{B}^{\mu \nu}+\mathcal{L}_{\mathrm{SM}}$,

and, with $D_{\mu}=\partial_{\mu}+i g_{D} Q_{D} \hat{V}_{\mu}$ being the gauge covariant derivative in obvious notation,

$\mathcal{L}_{2}=i \bar{\chi} \gamma^{\mu} D_{\mu} \chi-m_{D} \bar{\chi} \chi+\left(D_{\mu} S\right)^{\dagger}\left(D^{\mu} S\right)+\mu_{S}^{2} S^{\dagger} S-\lambda_{S}\left(S^{\dagger} S\right)^{2}$,

which describes the DM field $\chi{ }^{2}$ Note that here the DM is assumed to be a fermion (which does not couple directly to the SM) for definiteness but the detailed nature of the DM will not play any significant role in the discussion that follows below and is included here only for completeness but will be more important in our subsequent work [12]. This same piece of the action also describes the dark

\footnotetext{
${ }^{2}$ Additional terms may also be present in this piece of the Lagrangian to generate an additional Majorana mass for $\chi$ if desired.
} 
Higgs, $S{ }^{3}$ We will make the further assumption in our discussion below that $2 m_{D}>m_{V}$ such that $V$ must decay to $\mathrm{SM}$ fields, e.g., $e^{+} e^{-}$, so that this state may be observable at, e.g., HPS [13] but not at, e.g., LDMX [14] if one searches only for final states with missing energy/momentum. The KM in $\mathcal{L}_{1}$ is removed and the fields become canonically normalized via the standard field redefinitions (to lowest order in $\epsilon$ ), $\hat{B}_{\nu} \rightarrow B_{\nu}+\frac{\epsilon}{c_{W}} V_{\nu}$, $\hat{V}_{\nu} \rightarrow V_{\nu}$. After this transformation and mass mixing with the SM $Z$ generated via the vev of the SM Higgs, $H$, is accounted for (in the limit of small $m_{V}^{2} / m_{Z}^{2}$ as is relevant in the present case), the physical $V$ mass eigenstate couples to the $\mathrm{SM}$ fields as $\simeq e \epsilon Q_{e m}$. It should be noted that since $m_{V}=g_{D} v_{s}$ with $g_{D} \sim 0.1-0.3$ as being assumed here while $S$ has a mass $\sqrt{2 \lambda_{S}} v_{s}$ it is natural that $m_{S}>m_{V}$ since $g_{D}$ is small. Note that $S$ has no gaugeinvariant, renormalizable coupling to the Dirac fermion DM. This implies, in the absence of any mixing with the SM Higgs, that $S$ decays as $S \rightarrow V V^{*}, V^{*} \rightarrow e^{+} e^{-}$ and possibly more likely via the on-shell process $S \rightarrow V V$ and not directly to DM states. In the $S \rightarrow V V$ case, this decay is prompt as we are assuming $g_{D} \sim 0.1-0.3$; this will be important in the discussion that follows.

As well known the $H$ and $S$ Higgs fields can also mix due to their vevs through the standard quartic term

$$
\mathcal{L}_{H S}=\lambda_{H S} H^{\dagger} H S^{\dagger} S
$$

with the magnitude of $\lambda_{H S}$ usually and necessarily being fine-tuned to very small values (unlike what can happen by employing extra dimensions $[15,16]$ where this mixing can be naturally set to zero via a choice of boundary conditions) to meet phenomenological constraints arising from measurements of Higgs properties at the LHC. To get an idea of this stringent requirement which will be important in later discussions, we can compare the mixing-induced partial width $\Gamma(H \rightarrow S S+V V)$ to the present limit on the invisible Higgs branching fraction $B=\mathrm{BF}(H \rightarrow$ inv $) \lesssim$ $0.20-0.25$ [17]. To leading order in this mixing, the HSS coupling is just $\simeq \lambda_{H S} v_{H} / 2$ with $v_{H} \simeq 246 \mathrm{GeV}$. Some algebra then tells us that this branching fraction (BF) bound implies that $\left|\lambda_{H S}\right| \lesssim 4.7 \times 10^{-3} \sqrt{B / 0.1}$. (Note that a bound of similar magnitude can be obtained by requiring that the mass of $S$ dominantly arises from its own vev.) Since one finds that the $H-S$ mixing angle is then $\theta_{H S} \simeq-\lambda_{H S} v_{S} / 2 \lambda_{H} v_{H}$, this result further implies that $\left|\theta_{H S}\right| \lesssim 1.8 \times 10^{-4}\left(\frac{v_{s} / v_{H}}{10^{-2}}\right) \sqrt{B / 0.1}$. In the current analysis, except where noted, we will generally set the $\lambda_{H S}$ coupling to an extremely small value, corresponding to a very tight

\footnotetext{
${ }^{3}$ Here we will often write $S$ as $\left(v_{s}+S+i G\right) / \sqrt{2}$, where $G$ is the Goldstone boson eaten by the dark photon and $S$ now represents the remaining real scalar field.
}

mixing angle bound, so that its influence on the dark sector fields can generally be safely ignored. However, we will return to this very important issue later on below.

So far we have briefly summarized the usual development of the dark photon/KM scenario to establish notation and basic assumptions; now let us return to a discussion of the portal matter part of the action. We recall that these vectorlike portal matter fermion states must be unstable so that at least one path for their decay must exist. For the more "conventional" vectorlike fermions that are usually discussed such decay paths are provided by their mixing (via the vev of the SM Higgs) with the analogous SM states which carry the same QCD and QED quantum numbers. For portal matter states this mixing option does not occur as they also carry dark charges while the SM Higgs does not and so a gauge-invariant coupling of the required type is absent. We can, however, arrange something quite similar instead by employing the SM singlet dark Higgs [18]. Amongst the many a priori possibilities to consider at the renormalizable level, since the dark Higgs is a SM singlet, the pair of portal matter fields, $F_{1,2}$ (with $Q_{D}= \pm 1$ ) must be chosen to transform as one of the familiar set of vectorlike fermions (vectorlike fermion), either vectorlike quarks or vectorlike leptons, which are analogous to the representations already appearing in the SM, i.e.,

$$
(T, B)^{T}, \quad(N, E)^{T}, \quad T, B, E
$$

where the simplest (but not necessary) possibility is clearly that the portal matter are transformed just like one of the SM $S U(2)_{W}$ isosinglets, i.e., $T, B, E$. It is now easy to see that only these 5 particular choices allow for the new states to decay to SM fields at tree level since $S$ is a SM singlet. To determine how this specific mixing may be generated we imagine that $\left|Q_{D}(S)\right|=1$; then we can write a new gauge-invariant term in the Lagrangian given by

$$
\mathcal{L}_{\text {Portal }}=\lambda_{1 a} \bar{F}_{1 L} f_{R}^{a} S+\lambda_{2 a} \bar{F}_{2 L} f_{R}^{a} S^{\dagger}+\text { H.c. }
$$

with $L(R)$ the usual helicity projections and $f^{a}$ being the corresponding SM fermion of the same color and hypercharge (i.e., electric charge) with family label $a=1,2,3$. When $S$ gets a vev, this piece of the action will generate off-diagonal mass terms, symbolically of the form $X_{i}^{a}=$ $\lambda_{i}^{a} v_{s} / \sqrt{2}$, inducing a mixing between portal matter fields $F_{1,2}$ with those in the SM, $f^{a}$, and eventually between themselves. For ease of presentation, we again consider the most simple case where the mixing of the $F_{i}$ is dominantly with only one generation of the $f^{a}=f$ which, in the SM, obtains a mass from the usual Higgs mechanism, $m_{f}$. Such a possibility can be arranged, e.g., through the proper use of some flavor-dependent discrete symmetries as shown in Ref. [19]. Denoting this set of fermion fields in the original weak basis as $\mathcal{F}^{0 T}=\left(f^{0}, F_{1}^{0}, F_{2}^{0}\right)^{T}$, the general form of the various mass terms for these fermions can be written as $\overline{\mathcal{F}}_{L}^{0} \mathcal{M} \mathcal{F}_{R}^{0}+$ H.c. where 


$$
\mathcal{M}=\left(\begin{array}{ccc}
m_{f} & 0 & 0 \\
X_{1} & m_{1} & 0 \\
X_{2} & 0 & m_{2}
\end{array}\right)
$$

which can be diagonalized, as usual, via a biunitary transformation $M_{D}=U_{L} \mathcal{M} U_{R}^{\dagger}$, where $M_{D}$ is diagonal so that $\mathcal{F}_{L, R}=U_{L, R} \mathcal{F}_{L, R}^{0}$ are the mass eigenstates. Again, as usual, $U_{L}$ is determined via the relation $M_{D}^{2}=$ $U_{L} \mathcal{M} \mathcal{M}^{\dagger} U_{L}^{\dagger}$ while $U_{R}$ is similarly determined via $M_{D}^{2}=$ $U_{R} \mathcal{M}^{\dagger} \mathcal{M} U_{R}^{\dagger}$.

Before proceeding we need to say a few words about the values of the entries appearing in $\mathcal{M}$ (outside of the familiar $\left.m_{f}\right)$. As we will discuss in more detail below, the portal matter states $F_{1,2}$ can always be pair produced at the LHC in the same manner as are the more conventional vectorlike fermions that are usually discussed but which do not carry any dark charges. Thus we might semiquantitatively expect that $m_{1,2}(T, B) \sim 1-2 \mathrm{TeV}$ while $m_{1,2}(E) \sim 0.2-0.5 \mathrm{TeV}$ or so [20] based on the current null searches [10] at the LHC in which only SM particles are involved in the direct decay final state. (Of course, as we will stress below, these standard searches for vectorlike fermions are not directly applicable in the present case under study.) On the other hand, since $v_{s}$ is at most a few $\mathrm{GeV}$ as we are interested in $m_{V}=g_{D} v_{s} \lesssim 1 \mathrm{GeV}$ or so, we might then expect that roughly $\left|X_{1,2}\right| \sim 1-\mathrm{a}$ few $\mathrm{GeV}$ unless the $\lambda_{i}$ are not $\mathrm{O}(1)$.

With these typical values in mind we can straightforwardly obtain useful approximate analytic expressions for the elements of the matrices $U_{L, R}$ to lowest order in terms of the small set of four hierarchical ratios $\left|X_{i}\right|^{2} / m_{j}^{2}, i, j=$ $(1,2)$ as

$$
U_{L}=\left(\begin{array}{ccc}
1-m_{f}^{2}\left[X_{1}^{2} /\left(2 a_{1}^{2}\right)+X_{2}^{2} /\left(2 a_{2}^{2}\right)\right] & m_{f} X_{1}^{*} / a_{1} & m_{f} X_{2}^{*} / a_{2} \\
-m_{f} X_{1} / a_{1} & 1-m_{f}^{2} X_{1}^{2} /\left(2 a_{1}^{2}\right) & m_{2}^{2} X_{1} X_{2}^{*} /\left(\delta a_{1}\right) \\
-m_{f} X_{2} / a_{2} & -m_{1}^{2} X_{1}^{*} X_{2} /\left(\delta a_{2}\right) & 1-m_{f}^{2} X_{2}^{2} /\left(2 a_{2}^{2}\right)
\end{array}\right),
$$

where we have defined $X_{i}^{2}=\left|X_{i}\right|^{2}, a_{i}=m_{i}^{2}-m_{f}^{2}$ and $\delta=m_{1}^{2}-m_{2}^{2}$; correspondingly, we also obtain

$$
U_{R}=\left(\begin{array}{ccc}
1-\left[m_{1}^{2} X_{1}^{2} /\left(2 a_{1}^{2}\right)+m_{2}^{2} X_{2}^{2} /\left(2 a_{2}^{2}\right)\right] & m_{1} X_{1}^{*} / a_{1} & m_{2} X_{2}^{*} / a_{2} \\
-m_{1} X_{1} / a_{1} & 1-m_{1}^{2} X_{1}^{2} /\left(2 a_{1}^{2}\right) & -m_{1} m_{2} X_{1} X_{2}^{*} /\left(\delta a_{2}\right) \\
-m_{2} X_{2} / a_{2} & m_{1} m_{2} X_{1}^{*} X_{2} /\left(\delta a_{1}\right) & 1-m_{2}^{2} X_{2}^{2} /\left(2 a_{2}^{2}\right)
\end{array}\right) .
$$

Here we see that, quite generally, the size of the elements in $U_{R}$ involving the mixing of the vectorlike fermion with the SM fermion are significantly larger than the corresponding ones appearing in $U_{L}$. It is interesting to note that if we had instead chosen the portal matter fermions to be in $S U(2)_{W}$ doublets we would obtain the same results as above but with the roles of $U_{L}$ and $U_{R}$ simply interchanged. We note that in the corresponding case of the more canonical isosinglet vectorlike fermion that mixes with the SM fields via the usual Higgs vev, it is the $U_{L}$ matrix elements which are large and not the $U_{R}$ ones as is the case above. Here, since $U_{L}$ is close to the identity in the present case, as far as any mixing of the portal matter with the SM fields is concerned, this results in a further suppression of the possible portal matter decay into the familiar vectorlike fermion final states involving the SM gauge bosons and Higgs field making these modes essentially irrelevant, vanishing to leading order in the $m_{f} \rightarrow 0$ limit. The three eigenvalues of $M_{D}^{2}$ are approximately given to leading order in the above mass ratios by $m_{f}^{2}(1+\xi)$ and $m_{i}^{2}\left(1+\delta_{i}\right)$ with $\delta_{i} \simeq-X_{i}^{2} / a_{i}$ and

$$
\xi \simeq-\frac{X_{1}^{2} a_{2}+X_{2}^{2} a_{1}}{a_{1} a_{2}}=\delta_{1}+\delta_{2}
$$

which demonstrates that all of the mass shifts due to mixing are generally quite small. Note that all of the expressions above simplify significantly for the cases where $f \neq t$ in which case the $m_{f}^{2}$ terms can always be safely neglected in comparison to the $m_{i}^{2}$. These mixings will then allow the portal matter to decay only to final states which are completely dominated by the presence of the dark fields $V, S$ and not the usual SM fields $W, Z, H$.

\section{SURVEY OF PHENOMENOLOGICAL IMPLICATIONS}

Our first goal is to understand how significantly the mixing of the SM fermion, $f$, with the vectorlike fermion, $F_{i}$, alters the $f$ couplings to the dark photon, the SM and dark Higgs, as well as to the usual SM gauge bosons. Here we will work in the limit where $m_{V}^{2} / m_{Z}^{2} \ll 1$ so that in the original weak basis the part of the $V$ coupling to the SM due its $\mathrm{KM}$ with the hypercharge gauge boson is just $e \epsilon Q_{f}$ as noted above; note that such a coupling is now experienced by both the usual SM fields as well as by the portal matter ones since they also carry SM charges. Further note that in this limit of a small mass ratio, the dark photon does not pick up any additional couplings due to mass mixing with 
the $Z$ (i.e., the dark $Z$ scenario [21]). Suppressing Lorentz indices we may express this dark photon coupling in the notation above as e€ $Q_{f}\left(\overline{\mathcal{F}}_{L}^{0} \mathcal{F}_{L}^{0}+L \rightarrow R\right) \rightarrow e \epsilon Q_{f}\left(\overline{\mathcal{F}}_{L} \mathcal{F}_{L}+\right.$ $L \rightarrow R)$ as this part of the interaction is trivially insensitive to the mass mixing of $f$ with $F_{1,2}$. However, we recall that, unlike the SM fields, the portal matter themselves also have a direct coupling to $V, \sim g_{D}$, even when $\epsilon \rightarrow 0$; we can express this interaction in the form $g_{D}\left(\overline{\mathcal{F}}_{L}^{0} C \mathcal{F}_{L}^{0}+L \rightarrow R\right)$ where $C$ is the just the diagonal matrix

$$
C=\left(\begin{array}{ccc}
0 & 0 & 0 \\
0 & 1 & 0 \\
0 & 0 & -1
\end{array}\right) .
$$

In the mass eigenstate basis this interaction then just becomes $g_{D}\left(\overline{\mathcal{F}}_{L} C_{L} \mathcal{F}_{L}+L \rightarrow R\right)$ where we define $C_{L, R}=$ $U_{L, R} C U_{L, R}^{\dagger}$ with the elements of theses effective coupling matrices now given by $\left(C_{R}\right)_{i j}=U_{i 2}^{R} U_{j 2}^{R *}-U_{i 3}^{R} U_{j 3}^{R *}$ and similarly for $L \rightarrow R$ where $(i, j=1,2,3)$ corresponds to the mass eigenstates $f, F_{1}, F_{2}$, respectively. Some obvious but important things to note about these matrices include: (i) off-diagonal couplings to the dark photon between the $\mathrm{SM} f$ and the vectorlike fermion portal matter are generated allowing for decays such as $F_{1,2} \rightarrow f V$ with rates controlled by (the squares of) factors of order $m_{i} X_{i} / a_{i}$. (ii) $f$ picks up a mixing-induced diagonal coupling to the dark photon proportional to $g_{D}$ that is not vectorlike, i.e., parity violating, since $U_{L} \neq U_{R}$. Explicitly we find, using the matrices above that $\left(C_{R}\right)_{11}=m_{1}^{2} X_{1}^{2} / a_{1}^{2}-(1 \rightarrow 2)$ while $\left(C_{L}\right)_{11}=m_{f}^{2} X_{1}^{2} / a_{1}^{2}-(1 \rightarrow 2)$ and hence is smaller by factors of $m_{f}^{2} / m_{1,2}^{2}$ which are $\ll 1$ (except possibly in the case when $f=t$ but even there this factor is still likely quite small). We will return to the implications of these couplings in our discussion below. Note that whereas our single SM field picks up parity-violating interactions with the dark photon by mixing with portal matter, in other scenarios [21] this happens via the mixing of the dark photon with the SM $Z$ to that all of the SM fields would have now have parity-violating interactions.

The mixing of $f$ with the portal matter can also lead to alterations in the couplings of these fields to the SM $W, Z$ bosons. In the $Z$ boson case, since $f_{R}, F_{1,2 R}$ are all weak isosinglets, the rotation of these fields by the matrix $U_{R}$ has no impact on their $Z$ couplings; this is no longer true for the corresponding left-handed fields. In the original weak basis these fields couple as $\frac{g}{c_{W}}\left(T_{3 f} C_{Z}-x_{W} Q_{f} I\right)$ where $I$ is the $3 \times 3$ identity matrix, $x_{W}=\sin ^{2} \theta_{W}, T_{3 f}= \pm 1 / 2$ is the usual third component of the weak isospin for the SM field $f_{L}$ and $C_{Z}$ is just

$$
C_{Z}=\left(\begin{array}{lll}
1 & 0 & 0 \\
0 & 0 & 0 \\
0 & 0 & 0
\end{array}\right) .
$$

The resulting elements of the left-handed coupling matrix in the mass eigenstate basis then become $\frac{g}{c_{W}}\left(T_{3 f} U_{i 1}^{L} U_{j 1}^{L *}-\right.$ $\left.x_{W} Q_{f} \delta_{i j}\right)$ with $\delta_{i j}$ the usual Kronecker delta. There are again several simple but important things to note: (i) for the SM $f$, the quantity proportional to $T_{3 f}$ now differs from unity by a term of order $m_{f}^{2} X_{i}^{2} / a_{i}^{2} \ll 1$ even for the top quark. Thus to a good approximation the couplings of the SM fields to the $Z$ are left unaltered by the mixing with portal matter; a similar argument can be made for the $W$ as well since the same factor of $U_{i 1}^{L} U_{j 1}^{L *}$ will also appear there. (ii) Off-diagonal couplings are generated such that decays such as $F_{1,2} \rightarrow f Z$ (as well as $f^{\prime} W$ ) are now allowed but are controlled by (the squares of) factors of order $m_{f} X_{i} / a_{i}$. Note that these factors (squared) are smaller by $\sim m_{f}^{2} / m_{1,2}^{2} \ll 1$ than those controlling the decay $F_{1,2} \rightarrow$ $f V$ described above; a similar suppression is observed in the case of the $W$. Hence the portal matter decays into the dark photon (and $S$ as we will find below) will far dominate those into the conventionally sought $W, Z$ final state modes which vanish in the $m_{f} \rightarrow 0$ limit. The impact of this will be discussed further in more detail below.

Finally, we note that something similar happens when we consider the fermion couplings to the SM Higgs, $H .{ }^{4}$ In the weak basis this is just $\frac{\sqrt{2} m_{f}}{v_{H}} \overline{\mathcal{F}}_{L}^{0} C_{Z} \mathcal{F}_{R}^{0} H+$ H.c. using the notation above so that in the mass basis a coupling matrix, $C_{H}=U_{L} C_{Z} U_{R}^{\dagger}$, is produced whose elements are given by $\left(C_{H}\right)_{i j}=U_{i 1}^{L} U_{j 1}^{R *}$ and where we again see that the deviation of $\left(C_{H}\right)_{11}$ from unity is highly suppressed. Off-diagonal terms in $C_{H}$ allow for the decays $F_{1,2} \rightarrow f H$ but these are found to have suppressed (squared) couplings $\simeq m_{f}^{2} X_{i}^{2} / a_{1}^{2}$ similar to those for the $W, Z$ final states above so that these partial widths cannot remotely compete with those for $F_{1,2} \rightarrow f V$. This result should come as no surprise as this follows immediately from use of the Goldstone boson equivalence theorem [22]. In a similar manner, we can determine the corresponding coupling of the remaining real dark Higgs scalar, $S$, to the various fermions that are induced by the off-diagonal coupling above; we can express this result simply as $\beta_{i j}=\left[U_{i 2}^{L} \lambda_{1} / \sqrt{2}+U_{i 3}^{L} \lambda_{2} /\right.$ $\sqrt{2}] U_{j 1}^{R *}$.

\section{A. Leptonic portal matter}

We now turn to an examination of some sample implications of the above mixing and generated couplings for several choices of $f$; clearly the phenomenology will strongly depend upon the nature of the vectorlike fermion portal matter and what SM field(s) it mixes with via the dark Higgs. Here we will limit ourselves to a few simple

\footnotetext{
${ }^{4}$ We have set the $H-S$ mixing exactly to zero for this discussion due to the constraints mentioned above.
} 
examples, reminding ourselves that in a more complete theory the situation may be much more complex than what we consider here.

We first consider the case of a vectorlike lepton, i.e., $F=E$, so that $f=e$ above; in this case $U_{L} \simeq I$ (at least as far as the $e e$ and $e E_{1,2}$ elements are concerned) due to the small value of $m_{e}$ relative to all the relevant mass scales and so we can completely neglect left-handed mixing as far as SM fields are concerned in the rest of the present discussion. However, the right-handed mixing is still active so that, collecting the various pieces, e.g., the coupling of the electron to the dark photon is now given by

$\left[-e \epsilon \bar{e} \gamma_{\mu} e+g_{D}\left(C_{R}\right)_{11} \bar{e} \gamma_{\mu} P_{R} e\right] V^{\mu} \equiv-e \epsilon \bar{e} \gamma_{\mu}\left(v_{e}-a_{e} \gamma_{5}\right) e V^{\mu}$

where $v_{e}=1-y$ and $a_{e}=-y$ with $y=g_{D}\left(C_{R}\right)_{11} / 2 e \epsilon$. One might roughly expect that $\left(C_{R}\right)_{11} \simeq X_{i}^{2} / m_{i}^{2} \simeq 10^{-4}$ in the present case based on the arguments above assuming that $m_{E} \sim 200-300 \mathrm{GeV}$, so that, very roughly, $0.01 \lesssim$ $|y| \lesssim 0.5$ and thus the dark photon may now mediate a reasonably strong parity-violating interaction for the electron. This possibility may be probed [21] by a number of low-energy (i.e., low momentum transfer, $Q^{2} \lesssim m_{V}^{2}$ ) experiments such as in atomic parity violation (APV)[23] or in polarized electron-electron/electron-proton scattering [24,25]. In the case of APV, the amount of parity violation is quantified by a parameter $Q_{W}$, the weak charge, which in the $\mathrm{SM}$ is just $-(A-Z)+\left(1-4 x_{W}\right) Z \simeq-73.23(1)$ [26] for the case of Cesium $(Z=55, A=133)$ whereas the experimental value is $-72.62(43)$ [26]. Dark photon exchange in the present setup produces an additional contribution to $Q_{W}$ given by

$$
\Delta Q_{W}^{V}=\frac{-8 \sqrt{2} \pi \alpha \epsilon^{2}}{G_{F} m_{V}^{2}} y Z \frac{m_{V}^{2}}{Q^{2}+m_{V}^{2}},
$$

where $G_{F}$ is the Fermi constant and which is given numerically (with $Q^{2} \rightarrow 0$ ) by

$$
\Delta Q_{W}^{V}(C s) \simeq-1.22 y\left(\frac{100 \mathrm{MeV}}{m_{V}}\right)^{2}\left(\frac{\epsilon}{10^{-4}}\right)^{2},
$$

which must satisfy the constraint $-0.23 \leq \Delta Q_{W}^{V} \leq 1.45$ at 95\% C.L. to be consistent with the current experimental result. This is relatively easily done for values of $|y|$ in the above range of interest even for correspondingly respectable ranges in the values of both the parameters $m_{V}$ and $\epsilon$. The parameter space probed here is shown in the top panel of Fig. 1.

Polarized Moeller scattering [24,27], on the other hand, will offer a different probe of $y \neq 0$ as it leads to an apparent shift in the effective value of the weak mixing angle which can be written as
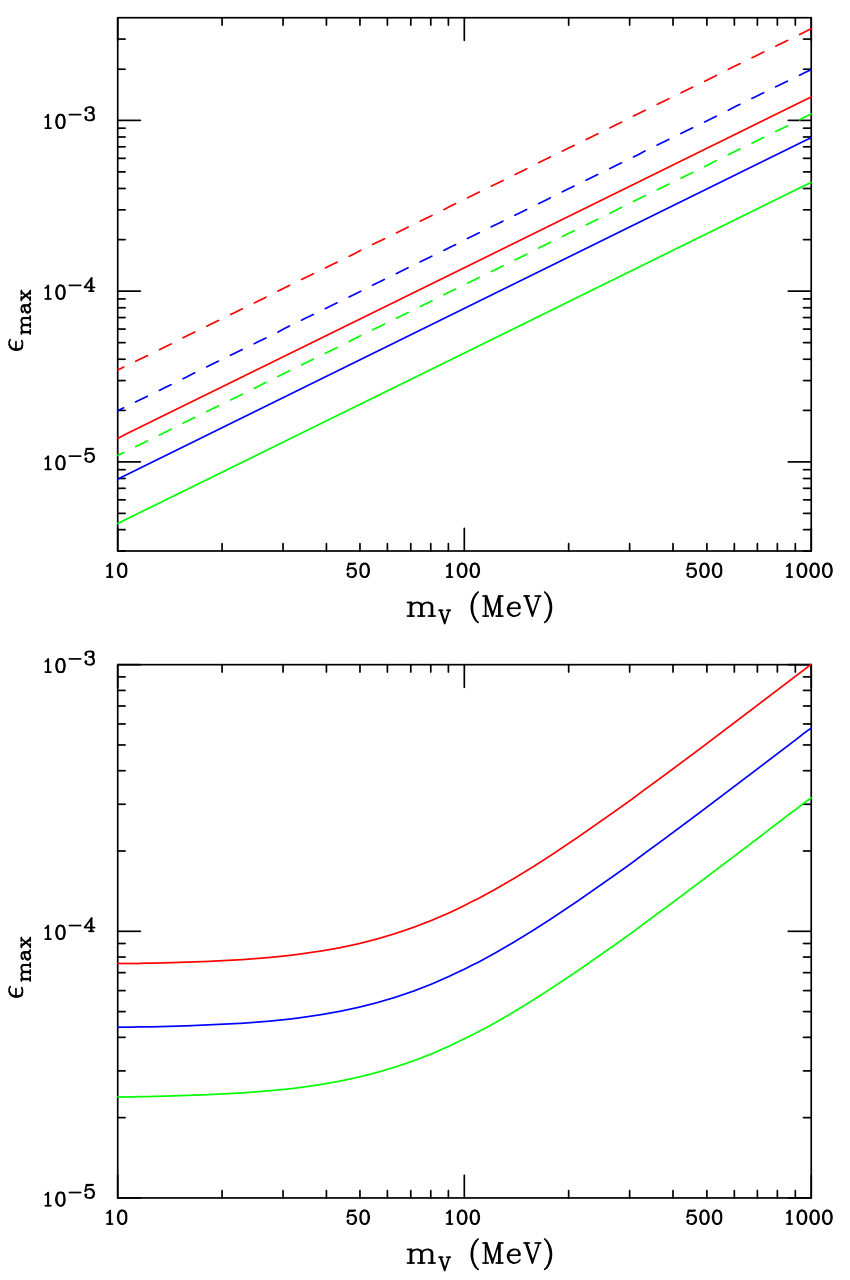

FIG. 1. (Top) Maximum value of $\epsilon$ as a function of $m_{V}$ allowed by the present APV data for positive (negative) values of $y$ as solid (dashed) lines. From top to bottom, $|y|=0.1,0.3$ or 1 has been assumed, respectively. (Bottom) Parameter space to be probed by the MOLLER experiment assuming the SM result is recovered. Here, again we see the maximum value of $\epsilon$ as a function of $m_{V}$ for, from top to bottom $|y|=0.1,0.3,1.0$, respectively.

$\Delta x_{W} \simeq 5.56 \times 10^{-3} y\left(\frac{100 \mathrm{MeV}}{m_{V}}\right)^{2}\left(\frac{\epsilon}{10^{-4}}\right)^{2} \frac{m_{V}^{2}}{\left\langle Q^{2}\right\rangle+m_{V}^{2}}$,

with $\left\langle Q^{2}\right\rangle$ being effective average value of $Q^{2}$ probed by the experiment which in this case is $\simeq(75 \mathrm{MeV})^{2}[23,24]$. Assuming that the SM value is realized at the end of the MOLLER experiment, this will result in a constraint $\left|\Delta x_{W}\right| \leq 5.6 \times 10^{-4}$. For $m_{V}=100(300) \mathrm{MeV}$ with $\epsilon=$ $10^{-4}$ this will imply a bound of $|y| \leq 0.16(1.2)$ which we again see can be easily satisfied. Quite generally, however, this experiment is observed to be able to probe a reasonable portion of the parameter space of this particular setup; of course a result in conflict with SM expectations would prove most interesting. The parameter space probed by 
MOLLER is shown in the lower panel of Fig. 1 assuming that the SM expectation is obtained; comparison with the APV shows that these two probes are complementary.

In the case where portal matter is muonlike instead of electronlike, i.e., $F=M$, one might ask if the added flexibility allowed by $y \neq 0$ could help explain the anomaly [28] in the muon's value of g-2 [26]; this question is easily addressed employing the general results given in [29]. There are actually several new contributions at the 1-loop level to this quantity beyond the ones usually appearing in dark photon models $[18,21]$. Ordinarily in such models the lone contributing diagram has a dark photon loop with the SM photon emitted off the internal muon line and where the dark photon coupling to the muon is both diagonal and purely vectorial; such a graph is usually simply suppressed by the small value of $\epsilon^{2}$ [21]. In the present scenario such a diagram exists but both vector and axial-vector couplings are now present. Generally the axial-vector contribution is found to add destructively with the one arising from the vector coupling making it less likely to explain the observation. Furthermore, this axial-vector contribution tends to be the numerically larger one so that the sum of both contributions is negative yielding the wrong sign from what is needed to explain the deviation from the SM. Employing the notation above we find the sum of these two contributions can be expressed as

$$
\begin{aligned}
\Delta g_{\mu}^{V_{1}}= & 2.32 \times 10^{-11}\left(\frac{\epsilon}{10^{-4}}\right)^{2} \int_{0}^{1} d x \\
& \times \frac{x^{2}(1-x)(1-y)^{2}-x(1-x)(4-x) y^{2}-2 x^{3} y^{2} r^{-1}}{x^{2}+r(1-x)}
\end{aligned}
$$

where $r=m_{V}^{2} / m_{\mu}^{2} \sim 1$ and explicitly shows the opposite sign contribution arising from the axial-vector coupling term. The magnitude of this integral is usually $\lesssim$ a few so that this overall contribution is quite small for our default parameter choices; for example, taking $r=1$ or larger one generally obtains rather small values for the integral and which can be of either sign depending upon the specific value of $y$. To obtain a more general understanding of this result, we can rewrite the expression above as

$$
\Delta g_{\mu}^{V_{1}}=10^{-11}\left(\frac{\epsilon}{10^{-4}}\right)^{2} R\left(y, m_{V}\right)
$$

where the quantity $R$ is shown in Fig. 2 as a function of $m_{V}$ for various values of $y$. Clearly, for our parameter choices $R$ is almost always negative and generally only yields at most a small overall contribution to the g-2 of the muon.

In addition to these "standard" dark photon contributions, there are now also a pair of 1-loop graphs with either $V$ or a dark Higgs loop [30] where the internal line is a portal matter state, $M_{i}$, and the couplings at both vertices are chiral. We note that in the conventional dark photon model, a dark Higgs contribution usually arises due to its

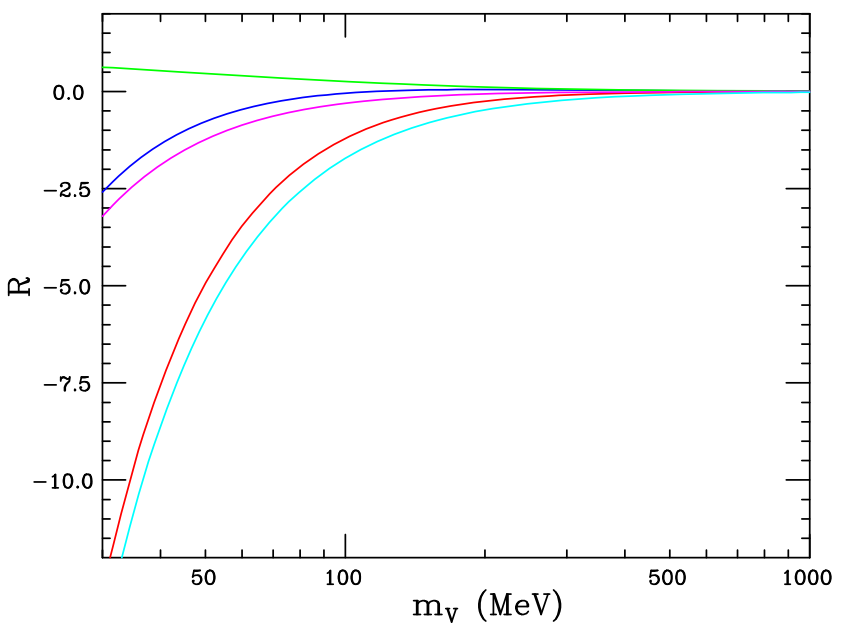

FIG. 2. The parameter $R$, as defined in the text, as a function of the $V$ mass $m_{V}$ assuming $y=-0.5(-0.25,0,0.25,0.5)$ corresponding to the red (blue, green, magenta, cyan) curves, respectively.

mixing with the SM Higgs and the muon resides on the internal line [30]. Here we find that the dominant dark Higgs contribution instead arises from the muon's mixing with the analogous portal matter state with the portal matter appearing on the internal fermion line. For these types of contributions the strong suppression is now seen to be the result of the rather small value of the ratio $m_{\mu}^{2} / m_{i}^{2}$ for the typical values of the portal matter masses that we might expect. In the scalar case we find (since $m_{S}^{2} \ll m_{i}^{2}$ ) that a positive result is obtained, i.e.,

$$
\Delta g_{\mu}^{S}=5.85 \times 10^{-10} \sum_{i} \frac{X_{i}^{2}}{v_{s}^{2}}\left(\frac{200 \mathrm{GeV}}{m_{i}}\right)^{2},
$$

which, since $\left|X_{i}\right| / v_{s} \sim 1 / 2$, is roughly an order of magnitude too small to make an important contribution unless the values of $m_{i}$ are significantly smaller than we might naively expect [20]. For the corresponding $V$ graph with portal matter on the internal line, we instead obtain

$\Delta g_{\mu}^{V_{2}}=-2.35 \times 10^{-4}\left(\frac{g_{D}}{0.1}\right)^{2}\left(\frac{100 \mathrm{MeV}}{m_{V}}\right)^{2} \sum_{i}\left(\frac{X_{i}^{2}}{m_{i}^{2}}\right)$,

where we have used the fact that $m_{i}^{2} \gg m_{V, \mu}^{2}$ and that $\left|\left(C_{R}\right)_{1 i}\right| \simeq\left|X_{i}\right| / m_{i}$ to leading order in the small mass ratios. Given the estimates above this contribution will also yield a negative result but only because most are as large as $\mathrm{O}\left(10^{-11}\right)$, similar to the first dark photon contribution obtained above.

Finally, we stress our overall result that in this current setup, $g-2$ does not constrain any of the model parameter space of interest to us particularly due to the multiple parameters that enter into the calculations and the corresponding numerical freedom that they represent. 
It is interesting to now very briefly consider how this singlet electron- or muonlike portal matter case differs from an alternative setup where the portal matter fields form the vectorlike lepton $S U(2)_{W}$ doublet $(N, E)^{T}$ [or $(N, M)^{T}$ ]. In the $Q=-1$ sector, all of the analyses presented in the previous discussion, with the interchange of left-handed and right-handed labels, essentially remain unaltered except that $y \rightarrow-y$. The presence of the $Q=0, N$, however, brings us something new. First, note that due to $S U(2)_{W}$ the weak states $N_{i}$ and $E_{i}$ will have essentially the same masses so that the $U_{L}$ matrices for both the $Q=0,-1$ sectors will be the same. This implies, in the limit that lepton masses can be neglected, that there is no new physics in the charged current sector coupling to $W^{ \pm}$. However, the mixing of $N$ with the ordinary left-handed $\mathrm{SM} \nu$ field now induces a coupling of this $\mathrm{SM} \nu$ field to the dark photon [31] of the form

$$
2 e \epsilon y\left(\bar{\nu} \gamma_{\mu} P_{L} \nu\right) V^{\mu},
$$

which may lead to observable effects in low energy neutrino experiments provided that the typical $\left\langle Q^{2}\right\rangle$ is not very much larger than $m_{V}^{2}$ [32]. We note in passing that when finite $m_{V}^{2} / m_{Z}^{2}$ corrections are included in the usual KM model (where portal matter effects are not considered), couplings to the neutrino are also induced as well but then these have a flavor universal strength suppressed by this very small mass ratio in comparison to ee $Q .{ }^{5}$ These couplings, where all of the neutrinos would be effected as opposed to a single flavor in the present case, are far smaller than the ones that can potentially be induced by mixing of the SM fields with the portal matter since $|y|$ can be sizable as discussed above. This possibility will be explored elsewhere [12].

\section{B. Hadronic portal matter}

Now let us turn our attention to our main emphasis, i.e., the perhaps more interesting case where the portal matter fields also carry color which to our knowledge has never been considered previously. Here we mainly focus on the specific example of the bottomlike singlet vectorlike quark, i.e., $F_{i}=B_{i}$, acting as the portal matter. While the case $F_{i}=T_{i}$ shares several features with this possibility, it has many unique aspects of its own which we will briefly discuss and contrast with the $F_{i}=B_{i}$ scenario below. For $F_{i}=B_{i}$, the general mixing/coupling analysis presented above remains applicable as $m_{b}^{2} \ll m_{1,2}^{2}$. Instead of flavor physics and low-energy phenomena, here we will be more interested in $B_{i}$ production and decay at the LHC especially as we anticipate that roughly $m_{1,2} \sim 1-2 \mathrm{TeV}$ in this case. ${ }^{6}$

\footnotetext{
${ }^{5}$ This possibility was also briefly discussed in [18].

${ }^{6}$ Of course, if there were additional flavor symmetries active in the quark sector, the implications on low energy phenomenology of such new states that might be involved in rare $B$ decays could also be very interesting.
}
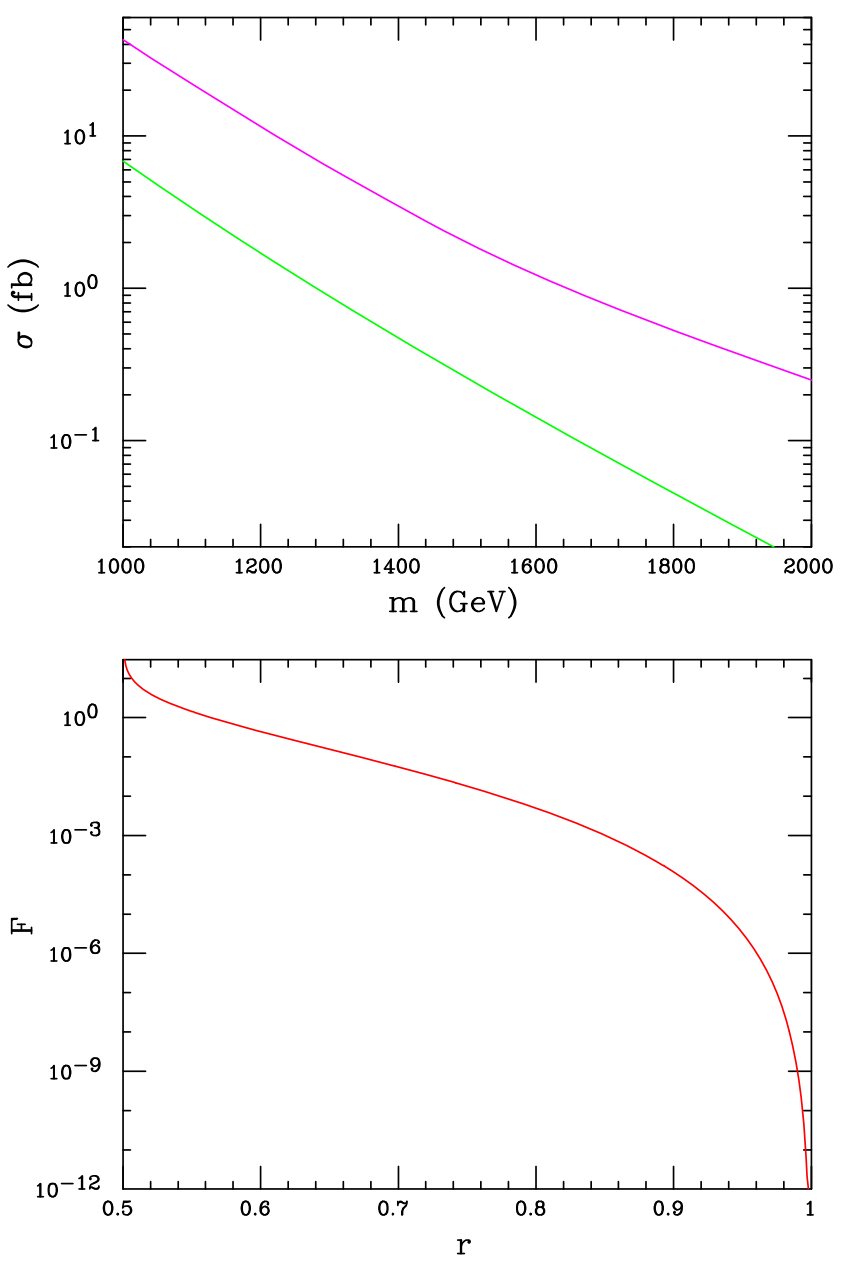

FIG. 3. (Top) $B \bar{B}$ (magenta) and $b$-squark (green) pairproduction cross section at $\sqrt{s}=13 \mathrm{TeV}$ as discussed in the text. (Bottom) Values of the 3-body phase space function $F$ for the decay $S \rightarrow V e^{+} e^{-}$as a function of $r=m_{V} / m_{S}$ as described in the text.

We first examine the standard QCD pair-production mechanism which is model independent except for the values of $m_{1,2}$. To set the scale for the overall event rate, note that at $\sqrt{s}=13 \mathrm{TeV}$ the production cross section for $\bar{B} B$ production at the LHC would be roughly $\simeq 42(2.0,0.60,0.25)$ fb for $m_{B}=1(1.5,1.8,2) \mathrm{TeV}$ [33], respectively, as shown in the top panel of Fig. 3. Generally, for this interesting mass range, this cross section is seen to scale roughly as $\sim m_{B}^{-7}$ so that values for other nearby masses can be easily obtained. This also implies that if $m_{B_{2}} / m_{B_{1}}=1.5(1.8)$ then $\sigma\left(B_{2}\right) / \sigma\left(B_{1}\right) \lesssim 0.06(0.02)$ so that almost the entire signal would arise from the lighter of the two states; we will mostly concentrate our analysis on the lighter of these two states below. As noted above (and as now applied specifically to this example), one easily finds that the partial decay widths for the conventional modes such as $B_{i} \rightarrow b Z, b H$ and $B \rightarrow t W$ are quite highly suppressed in comparison to the partial widths for decays to the $b V, b S$ final states, vanishing completely in 
lowest order in the $m_{b} \rightarrow 0$ limit. This implies that the ordinary modes usually employed at the LHC in the searches for vectorlike quarks are inoperable in this case as noted previously.

How do the partial widths for the two $B_{i} \rightarrow b V, b S$ decay modes compare? We first recall that both the $B_{i}$ are likely to have qualitatively comparable masses such that they satisfy $m_{1,2}^{2} \gg m_{b}^{2}, m_{S, V}^{2}$ since both $m_{V, S}$ are $\sim$ a few $\mathrm{GeV}$ at most. In the limit where we can neglect all of the light particle masses appearing in the final state we find that the partial widths for both the $V, S$ modes are identical (again this is due to the equivalence theorem [22]) and are given in this small mixing angle, small masssquared ratio limit by

$$
\Gamma\left(B_{i} \rightarrow b V, b S\right)=\frac{\lambda_{i}^{2} m_{i}}{32 \pi},
$$

where the $\lambda_{i}$ have been defined above and are nominally of $\mathrm{O}(1)$. These decays are clearly prompt even for substantially smaller values of the $\lambda_{i}$ 's. It is important to remind ourselves of the lifetimes of the $S, V$ states that appear here as $B_{i}$ decay products. (The decay of the heavier $B_{2}$ state into the lighter $B_{1}$ plus $V$ or $S$ is found to be suppressed in rate by factors of order $\sim v_{s}^{2} / m_{2}^{2}$ [12] in comparison to that to the $b V, S$ final state.) The well-known total decay width $[6,7]$ for $V$ can be written in terms of its partial width into the $e^{+} e^{-}$final state and the branching fraction for this mode, $B_{e}\left(m_{V}\right)$, as (taking here the $m_{e}^{2} / m_{V}^{2} \rightarrow 0$ limit for simplicity given the $V$ mass range of interest below)

$$
\begin{aligned}
\Gamma(V \rightarrow \text { all })= & \frac{\alpha \epsilon^{2} m_{V}}{3 B_{e}}=2.432 \times 10^{-9}\left(\frac{\epsilon}{10^{-4}}\right)^{2}\left(\frac{m_{V}}{100 \mathrm{MeV}}\right) \\
& \times \frac{1}{B_{e}} \mathrm{MeV},
\end{aligned}
$$

which corresponds to an unboosted decay length for $V$ of

$$
c \tau \simeq 81.44\left(\frac{10^{-4}}{\epsilon}\right)^{2}\left(\frac{100 \mathrm{MeV}}{m_{V}}\right) B_{e} \mu m .
$$

Note that for the corresponding $V$ decay to pairs of muons above threshold, the above electronic partial width must be scaled by a factor of $\beta_{\mu}\left(3-\beta_{\mu}^{2}\right) / 2$ where $\beta_{\mu}^{2}=1-4 m_{\mu}^{2} / m_{V}^{2}$. In our numerical analyses, in order to obtain the relevant value of $B_{e}$, we have employed the $e^{+} e^{-} \rightarrow$ hadrons results and corresponding treatment as described in Ref. [34].

Now recall that $m_{V} / m_{S}=g_{D} / \sqrt{2 \lambda_{S}}$ so it is very likely that $m_{S}>m_{V}$ and we will assume that this is true in what follows. When $m_{S}>2 m_{V}$, then the dominant $S$ decay is naturally $S \rightarrow V V$ via the vev, $v_{s}$, with a partial width given by $^{7}$

\footnotetext{
${ }^{7}$ Recall that in the present setup, $S$ has no tree-level allowed decay to DM assuming that such a mode is even kinematically open.
}

$\Gamma(S \rightarrow V V)=\frac{g_{D}^{2} m_{S}}{128 \pi} \frac{1}{x_{V}}\left(1-4 x_{V}\right)^{1 / 2}\left(1-4 x_{V}+12 x_{V}^{2}\right)$,

where $x_{V}=m_{V}^{2} / m_{S}^{2}$ and this is also seen to be a prompt decay, even near threshold. If instead we have that $m_{V}<m_{S}<2 m_{V}$, we obtain a partial width of

$$
\begin{aligned}
\Gamma\left(S \rightarrow V e^{+} e^{-}\right)= & \frac{g_{D}^{2} \alpha \epsilon^{2} m_{S}}{96 \pi^{2}} F(r)=7.7 \times 10^{-16} m_{S}\left(\frac{g_{D}}{0.1}\right)^{2} \\
& \times\left(\frac{\epsilon}{10^{-4}}\right)^{2} F(r),
\end{aligned}
$$

where here $1 / 2<r=m_{V} / m_{S}=\sqrt{x}_{V}<1$ and $F$ is a wellknown function [35] that ranges over many orders of magnitude and whose behavior we display in the lower panel of Fig. 3. We note, for example, that for $r=$ $0.6(0.7,0.8,0.9)$ one finds that $F=0.443\left(5.55 \times 10^{-2}\right.$, $\left.4.97 \times 10^{-3}, 1.18 \times 10^{-4}\right)$, respectively, so that $F$ falls off quite rapidly with increasing $r$, and this can lead to a very substantial width suppression/lifetime lengthening due to phase space. Of course, very close to the $V V$ threshold finite $V$ width effects will become important here but as noted above the $V$ total width itself is quite small due to the appearance of the overall $\epsilon^{2}$ suppression factor. Since it is clear that this $S \rightarrow V e^{+} e^{-}$partial width is extremely small we must take some care that $S$ has no other possible decay modes than may compete, e.g., through the very suppressed mixing with the SM Higgs via a tiny, but nonzero value of $\lambda_{H S}$ as discussed above. For example, the $H-S$ mixinginduced partial width for $S \rightarrow l^{+} l^{-}$is given by

$\Gamma\left(S \rightarrow l^{+} l^{-}\right) \simeq \frac{G_{F} m_{l}^{2} m_{S}}{4 \sqrt{2} \pi} \beta_{l}^{3} \theta_{H S}^{2} \lesssim 2.4 \times 10^{-16} m_{S}$,

where in the last step we consider the case of $l=\mu$, have set the phase space factor $\beta_{l} \rightarrow 1$, employed the upper limit on the value of $\theta_{H S}$ discussed above with $v_{s} / v_{H}=10^{-2}$ and assumed an invisible Higgs branching fraction of $B_{\text {inv }}=0.1$. (The corresponding partial width into $e^{+} e^{-}$is smaller by roughly a factor of $\sim 4.3 \times 10^{4}$.) Here we see that if $S$ lies above the $\mu^{+} \mu^{-}$threshold and we saturate the LHC bound on the $H-S$ mixing coming from SM Higgs decay data then the rate for the $S \rightarrow \mu^{+} \mu^{-}$decay may be comparable to or even dominate over that for $V e^{+} e^{-}$especially since the function $F$ is potentially very small. Here we will assume that this is not the case, e.g., we will always assume that the mixing of $S$ with the SM Higgs is so small that it has no influence upon how $S$ decays; we proceed with this caveat kept in mind. Inserting typical values into the above expression for the $S \rightarrow V e^{+} e^{-}$ partial width leads to an unboosted $S$ decay length for masses in the relevant interval of

$c \tau \simeq 170.8\left(\frac{0.1}{g_{D}}\right)^{2}\left(\frac{10^{-4}}{\epsilon}\right)^{2}\left(\frac{150 \mathrm{MeV}}{m_{S}}\right) \frac{1}{F(r)} \mathrm{cm}$. 
We note that extremely long proper lifetimes for $S$, of order $1 \mathrm{~s}$ or more, which could result in some parameter space regions, are likely excluded by cosmological considerations and particularly by the bounds that arise from nucleosynthesis constraints [36].

From these estimates it would seem that bottomlike portal matter (generically $B$ for simplicity) pair production at the LHC would produce quite unusual events in certain phase space regions, i.e., the apparent signatures for $B$ pair production depend critically upon where one sits in the model parameter space. Perhaps the simplest situation is where both the $B$ and $\bar{B}$ each promptly decay into a dark photon, i.e., the $b \bar{b} V V$ final state. Since the $B$ 's are presumed heavy and relatively slow their decay will yield two, high- $p_{T}, b$-jets (that can act as the trigger) which are generally not back to back. Both $V$ 's will be very highly boosted and also will likely not be back to back, each sharing the parent $B$ rest energy with the associated $b$-jet, so that the resulting boost is roughly given by $\gamma_{V} \simeq$ $m_{B} / 2 m_{V} \sim 5000(1000)$ if $m_{V}=100(500) \mathrm{MeV}$ and $m_{B}=1 \mathrm{TeV}$; the boost is, of course, somewhat larger if $B$ is even more massive. To get a rough idea of the resulting decay properties and boosted decay lengths $\left(d=\gamma_{V} \beta c \tau\right)$ of the dark photon within the interesting parameter space region we perform the following scan: (i) we take $\epsilon$ to lie in the range $10^{-5}-10^{-3.5}$ while, independently, (ii) $m_{V}$ to lie in the range $10^{1.5}-10^{3} \mathrm{MeV}$. Specific values in these ranges are chosen employing log priors. Once $m_{V}$ is known we can then estimate the value of $B_{e}\left(m_{V}\right)$ [34] and hence determine the quantity of interest, $d$, using the equation above. We note that the low-mass end of this range where $\epsilon$ is simultaneously chosen to also be at the lower end of its range is somewhat disfavored by existing experiments [5]. We do not account for this as part of the scan but point out that it is in this region that the longest $V$ lifetimes (and corresponding large boosts) are frequently obtained. Given these numerical assumptions and caveats, the result of this scan for $10^{7}$ generated model points is shown as the histogram in the top panel of Fig. 4. For these assumed parameter ranges, the peak of this distribution is seen to lie roughly at $d \sim 0.7 \mathrm{~m}$ but the distribution of possible values for $d$ is observed to have quite long, slightly asymmetric tails going down to values of $d \sim 2 \mathrm{~mm}$ and up to $d \sim 400 \mathrm{~m}$. In all cases $m_{B}=1 \mathrm{TeV}$ has been assumed here; larger mass values will lead to correspondingly increased boosts but only by a small factor. Of course, any given model point in this parameter space will realize only a specific $d$ value within this range. We note the trivial observation that for a fixed value of $d$, the two $V$ 's in the final state can generally decay at different distances from the IP and these may even occur in different LHC detector elements.

For those regions of the parameter space where both $V$ decays will very likely occur long after they leave the detector, such events would essentially appear (incorrectly) as having missing $E_{T}$ (MET) in addition to the two $b$-jets.
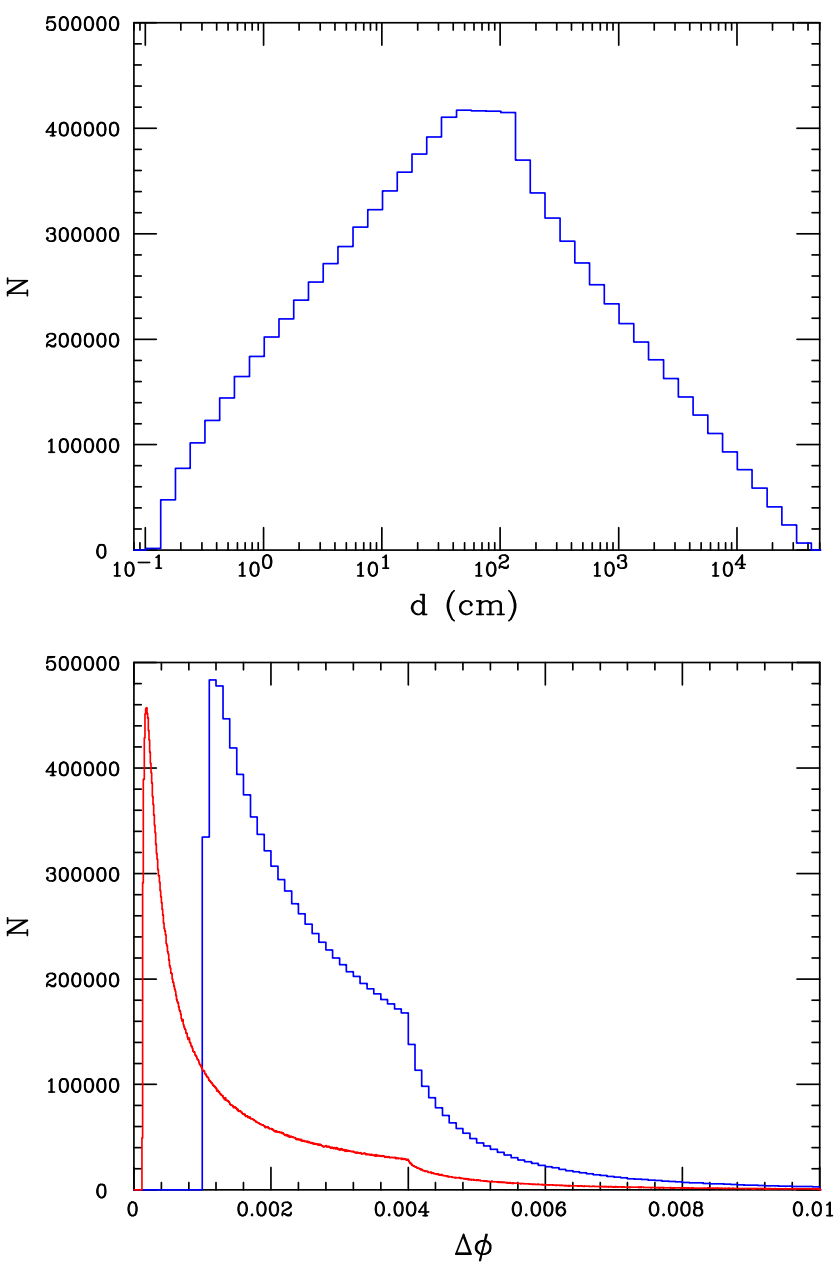

FIG. 4. (Top) Boosted decay length $(d)$ distribution of the dark photon from the decay $B \rightarrow b V$ assuming that $m_{B}=1 \mathrm{TeV}$ as described in the text. (Bottom) Boosted leptonic opening angle distributions, averaged over the model parameter space, for the boosted $V \rightarrow e^{+} e^{-}$(red) and $V \rightarrow \mu^{+} \mu^{-}$(blue) decay modes.

This is a "conventional" signature that is essentially covered by the usual SUSY direct $b$-squark decay to bottom plus the LSP searches [37], albeit with somewhat different production cross sections and acceptances. ${ }^{8}$ The present null results for these $b$-squark searches in the limit of a massless neutralino (which most closely corresponds to the present possibility under discussion) certainly would imply that $m_{1,2}>1-1.5 \mathrm{TeV}$ in such cases. If we had instead considered the toplike portal matter, $T$, which would similarly decay to a $t V$ final state with a very long-lived $V$, such signatures would then be covered by the analogous top squark searches leading to somewhat similar, but slightly weaker, mass constraints on

\footnotetext{
${ }^{8}$ Note that the total $b$-squark pair-production cross section in this mass range for decoupled gluinos is roughly a factor of 6-10 smaller than is that for $B \bar{B}$ pairs $[38,39]$ as shown in the upper panel of Fig. 3.
} 
the portal matter due to the reduced efficiency of top reconstruction. Of course, if a secondary, "far" detector were to be available at an appropriate distance, such as MATHUSLA [40] or FASER [41], a respectable fraction of these long-lived particle decays might be captured and studied.

Perhaps more interesting are events where one or both of the $V$ 's decay before/inside the calorimeter/muon system after leaving no tracks in the inner part of the detector. If only one of the $V$ 's decay after leaving the detector, the event will still appear as two $b$-jets plus MET but with additional activity due to the other $V$ now decaying inside the detector. Obviously, if both $V$ 's decay inside the detector there will be no MET signature but the high- $p_{T}$ $b$-jets can still provide the necessary trigger. This makes the predictions of the current setup rather unique (due to the very large boosts as we will find below). If both/either $V$ decays into SM particles (e.g., $e^{+} e^{-}, \mu^{+} \mu^{-}, \pi^{+} \pi^{-}$) inside the detector it will appear as a long-lived/displaced lepton jet [42] and this has frequently been discussed as a dark sector signature (as it is here) although generally in kinematic regimes with significantly smaller boost values and without the $b$-tagged trigger jets. Searches for such lepton-jet final states within the context of specific signals models have been performed by both ATLAS and CMS at the LHC [43,44] with null results. ${ }^{9}$ An important observation is that for this decay mode, the opening angle between the $V$ decay products will be extremely small due to the large boost that the $V$ experiences from the parent $B$ decay. Estimates of these opening angles are shown in the lower panel of Fig. 4 for the both $e^{+} e^{-}$and $\mu^{+} \mu^{-}$final states which represented the parameter space weighted averages for these expectations (and not those associated with a specific parameter choice). For the $\mu^{+} \mu^{-}$final state case we added the additional constrain that $m_{V} \geq 250 \mathrm{MeV}$ as part of the scan while $m_{e}=0$ has been assumed throughout.

Since displaced lepton jets decaying at different depths in the detector are somewhat challenging to simulate with DELPHES [42,45], we can obtain an (albeit extremely crude) estimate for the largest possible rate for such events by assuming that, e.g., ATLAS is a perfect detector of radius $\sim 7 \mathrm{~m}$ with $100 \%$ acceptance and efficiency so that any $V \rightarrow$ lepton-jet events produced inside this detector volume are observed. Assuming an integrated luminosity of $100 \mathrm{fb}^{-1}$ for purposes of demonstration, and knowing the $B \bar{B}$ cross section as a function of the $B$ mass from above we know the total number of $B$ 's produced, all of which decay to $V$ or $S$ with an equal branching fraction. For any given

\footnotetext{
${ }^{9}$ Note that the corresponding appearance of prompt lepton jets are not very likely for the $V, S$ final states in the current setup due to the rather large boosts that are generally expected here. However, such searches may still play important roles in certain corners of the parameter space.
}

value of the boosted decay length, $d$, (employing the range as given in Fig. 4 and determined from our parameter space scan described above) we can determine the probability that $V$ will decay before reaching the $7 \mathrm{~m}$ edge of the detector, correcting for the different values of $m_{1}$. From this information, we then know the maximum number of single lepton-jet events that might be observed under these extremely optimistic assumptions of perfect efficiency (here assuming both the $b V$ and $b S$ final states produce at least one lepton jet) as a function of $d$. The result of this very simple calculation, which is clearly a sizable overestimate of reality, is shown in the upper panel of Fig. 5. From this result we can argue that for values of $d$
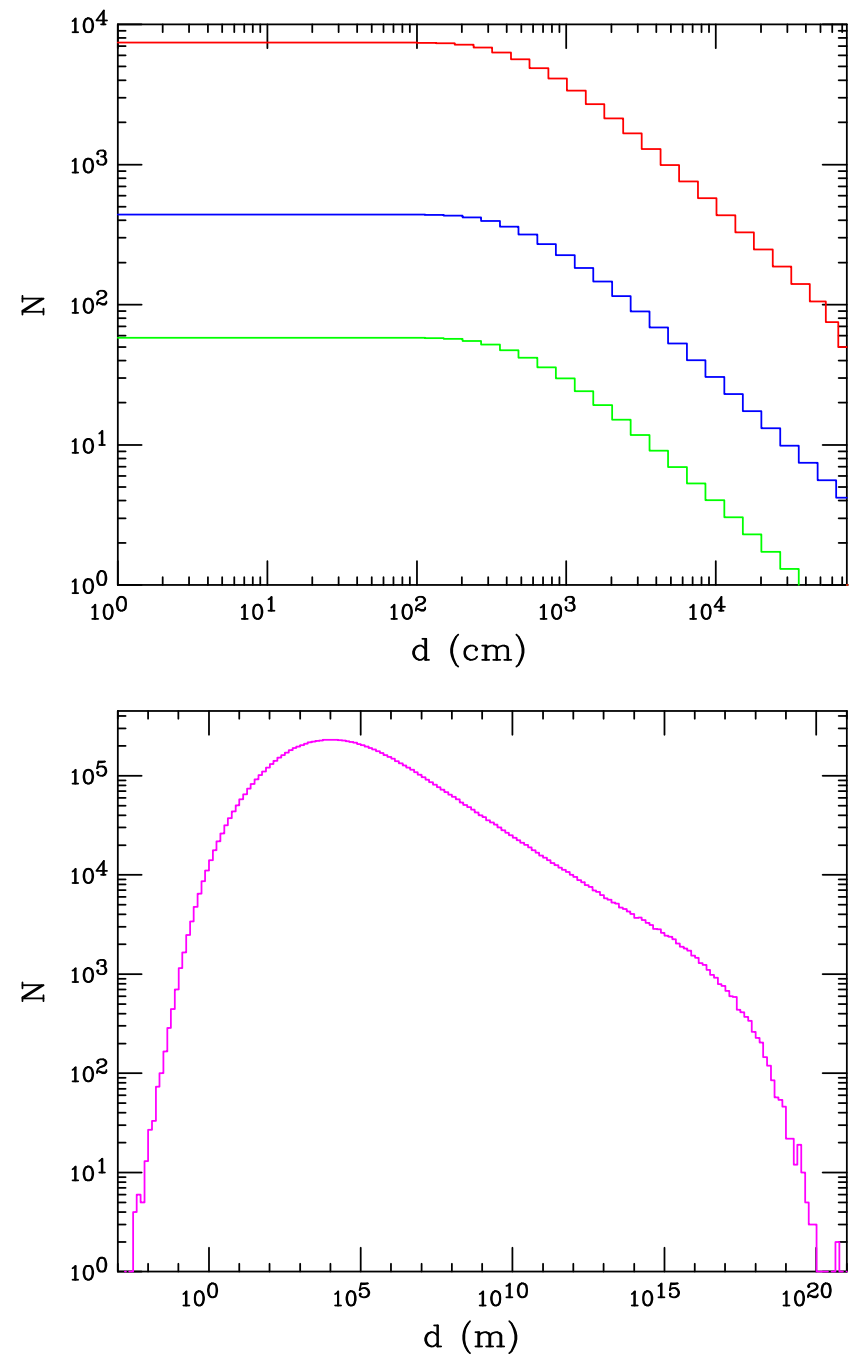

FIG. 5. (Top) Event rate estimate for the number of single lepton jets for the idealized ATLAS detector as discussed in the text assuming an integrated luminosity of $100 \mathrm{fb}^{-1}$. From top to bottom the histograms assume that $m_{1}=1,1.5,2 \mathrm{TeV}$, respectively. (Bottom) Boosted decay length $(d)$ distribution of the $S$ from the decay $B \rightarrow b S$ assuming that $m_{B}=1 \mathrm{TeV}$ as described in the text assuming $m_{V}<m_{S}<2 m_{V}$. Note that values of $d>10^{12} \mathrm{~m}$ or so are likely excluded by consideration of cosmological and nucleosynthesis constraints. 
below $\sim 10$ 's of meters lepton jets from the decay of $B$-like portal matter in the mass range of interest may likely be observable with the Run II data set. Of course a much more realistic analysis of this possibility is clearly extremely necessary.

When the decay $B \rightarrow b S$ occurs the critical issue is whether or not the bound $1<m_{S} / m_{V}<2$ is satisfied; although this appears to be a small region of the parameter space, it is the one most analogous to that realized in the SM for the Higgs and $W^{ \pm}$so we need to take this mass range possibility quite seriously. If the mass ratio does lie in this range, when $S$ receives a boost of $\sim 10^{3}-10^{4}$ from the original $B$, the boosted decay length of $S$ can easily exceed $\sim 10-100 \mathrm{~km}$ as its unboosted decay length is typically $\sim 2 F^{-1} \mathrm{~m}$ as discussed above. This result is shown in the lower panel of Fig. 5 which makes use of the previous parameter space scan and, in addition, now includes a scan over values of $1<m_{S} / m_{V}<2$ with a flat prior. Here the generally small value of the function $F(r)$ plays a rather significant role in increasing both the $S$ lifetime and the corresponding boosted decay length. In such a situation it is quite likely that the $V$ 's resulting from the $S$ decay will themselves decay to SM particles very far outside the detector. In such cases we return to the two $b$-jet plus MET signature as previously discussed and which yields a bound of $m_{1,2}>1-1.5 \mathrm{TeV}$ or more. We note that if $H-S$ mixing is important at the level discussed above, and if $S \rightarrow \mu^{+} \mu^{-}$is kinematically open, then its unboosted decay length will be greater than $\sim 0.55 \mathrm{~m}$; such values would still lead to quite long boosted decay lengths and result in the likely decay of $S$ outside of the detector much of the time.

When $m_{S}>2 m_{V}$, on the other hand, the $S \rightarrow V V$ decay is prompt resulting in a two $b$-jet plus $4 V$ final state with the two pairs of $V$ 's from each $S$ being strongly columnated due to the large boost. Since the $V$ 's in such a case result from a secondary decay in the chain and their preboost velocities can be oriented arbitrarily (with a flat distribution with respect to the motion of the $S$ since it is spin-0), their actual boosts will be somewhat reduced. In this case the secondary $V$ boosted decay lengths, shown in the top panel of Fig. 6, will be somewhat shorter than when the $V$ arises directly from $B \rightarrow b V$. (To obtain these numerical results we have assumed that $2 \leq m_{V} / m_{S} \leq 5$ with a flat prior.) Note that some of the $V$ decays in this parameter space region may now appear as prompt unlike when $B \rightarrow V$ directly. This $d$ distribution is found to display a peak value of $\sim 30 \mathrm{~cm}$ but, again, with rather long tails, extending below $\sim 1 \mathrm{~mm}$ at the lower end. The two $V$ 's from a given $S$ will likely not decay at the same time or possibly even in the same region within the detector since the ratio of the distinct boosts that the two $V$ 's receive from the $B \rightarrow S \rightarrow$ $V V$ decay chain can be $\mathrm{O}(1)$, this again due to the random
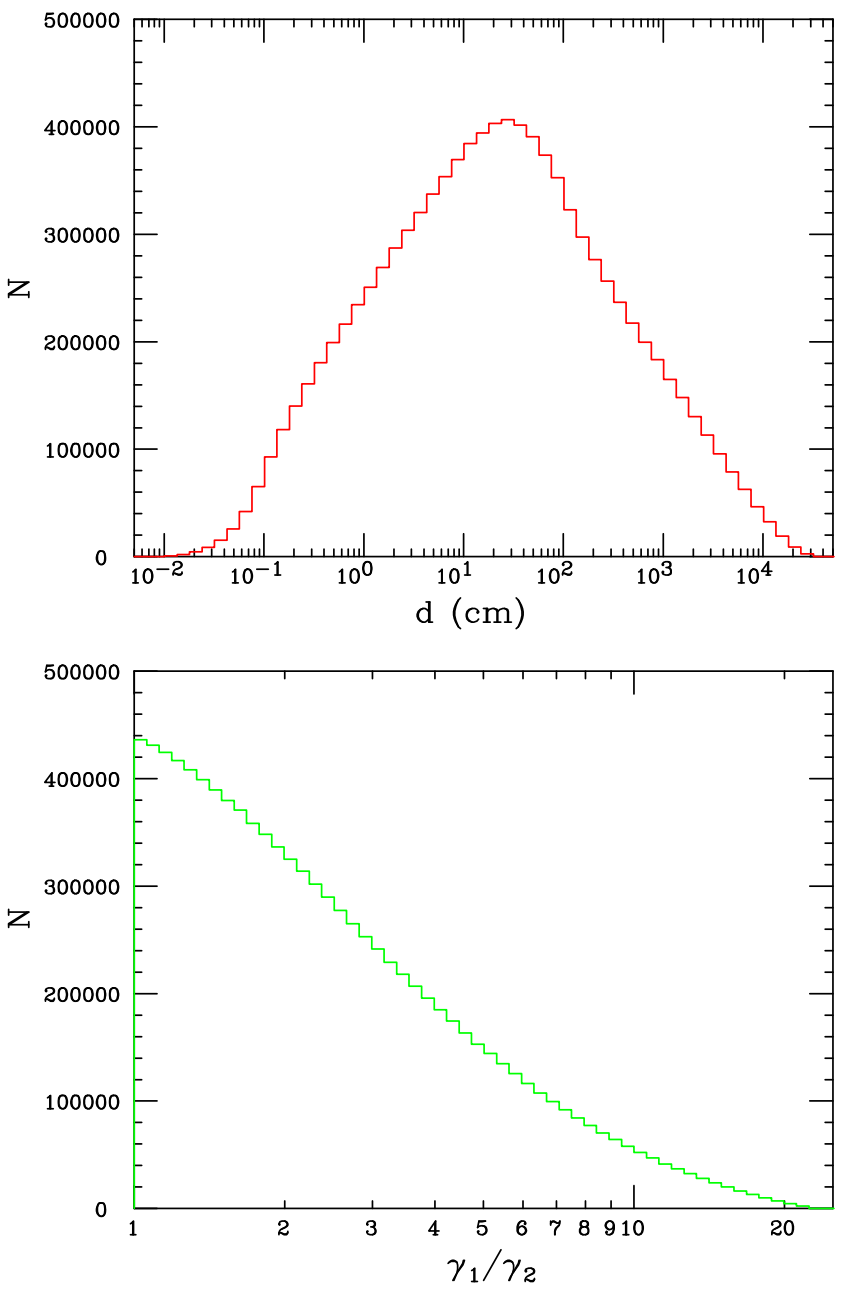

FIG. 6. (Top) Boosted decay length $(d)$ distribution of the $V$ arising from the decay chain $B \rightarrow b S, S \rightarrow V V$ assuming that $m_{B}=1 \mathrm{TeV}$ as described in the text. (Bottom) Ratio of the boosts of the two $V$ 's produced from a single initial $B$ (or $\bar{B}$ ) and the $B \rightarrow b S, S \rightarrow V V$ decay chain. $V_{1}$ is always defined to be the state with the larger boost.

orientation of the produced $V$ relative to direction of the moving $S$. The lower panel of Fig. 6 shows the ratio of these two boosts where $V_{1}$ is always defined as the one obtaining the larger boost in this decay chain. We see that while a ratio of unity is the most likely value, there is a large region of this model space where this ratio is significantly greater than unity. On average, employing the same scan results as above, we find that the opening angle between the two $V$ 's in the detector is generally still quite small as seen in Fig. 7. It is to be remembered that this result represents the weighted distribution for this opening angle averaged over the model parameter space and is not that corresponding to a specifically chosen parameter space point.

In addition to $B \bar{B}$ pair production, we briefly note that single $B$ associated production with either $V / S$ can also occur at a significant rate for $\lambda_{i}$ being $\mathrm{O}(1)$ with a lowest order cross section that is given by (in the limit $m_{b, V, S}^{2} \rightarrow 0$ ) 


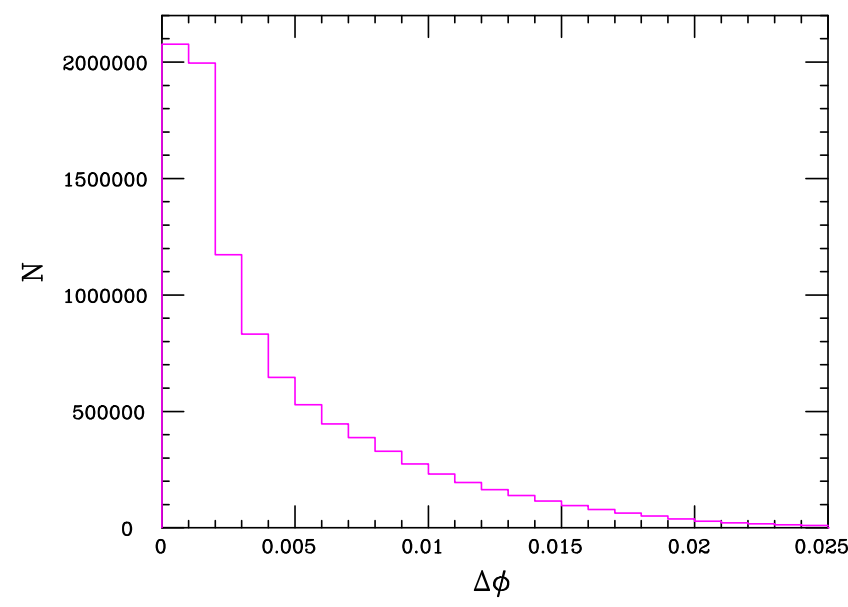

FIG. 7. Distribution of the opening angle between the two $V$ 's in $S$ decay, $\Delta \phi$, as described in the text.

$$
\begin{aligned}
\frac{d \sigma}{d \hat{t}}= & \frac{\lambda_{i}^{2} \alpha_{s}}{48 \hat{s}^{2}}\left[\frac{(\hat{s}+\hat{t})^{2}}{-\hat{s} t^{\prime}}+m_{i}^{2}(\hat{s}+\hat{t})\left(\frac{1}{\hat{s}^{2}}+\frac{1}{t^{\prime 2}}\right)\right. \\
& \left.-\frac{2 m_{i}^{2} \hat{t}}{t^{\prime}}\left(\frac{2}{t^{\prime}}+\frac{1}{s}\right)\right],
\end{aligned}
$$

where $t^{\prime}=\hat{t}-m_{i}^{2}$; note that in this limit, the equivalence theorem [22] tells us that production with either $S$ or $V$ yields the same cross section. As can be seen from this expression, the single-production process occurs via $g b$ fusion $\left(\rightarrow B_{i} V / S\right)$ with virtual $b\left(B_{i}\right)$ exchanges in the $s(t)$ channels. We note that in the corresponding case where $F_{i}=T_{i}$ such a production mechanism is absent since to a good approximation there are no tops in the proton. The value of this associated production cross section at the $\sqrt{s}=13 \mathrm{TeV}$ LHC is shown in the top panel of Fig. 8 as a function of the $B_{i}$ mass for different $p_{T}$ cuts on $S, V$ and as a function of the cut $p_{T}^{\min }(S, V)$ for different choices of the $B_{i}$ mass; in all cases an additional cut requiring a central event, $\left|\eta_{V, S}\right|<2.5$, has been applied. This associated production process, as in the case of $B \bar{B}$ production, leads to a very highly boosted $V, S$ arising from the $B$ decay itself. However, the associated $V, S$, which is also produced in this process, generally arrives with lower $p_{T}$ and at larger rapidities, as can be seen in the lower panel of Fig. 8 and in Fig. 9. Thus for fixed $p_{T}$ this $V, S$ is likely to experience a somewhat smaller boost especially at the smaller rapidities, $\left|\eta_{V, S}\right| \rightarrow 0$, in comparison to those produced in the $B$ decay. In particular we see that, for the range of masses of interest to us, almost all the events are found to lie in the region $\left|\eta_{V, S}\right| \gtrsim 0.5-1$. Such events can be triggered on by the appearance of the single high- $p_{T} b$-jet which now appears in the final state. In the case where $V / S$ decay outside the detector the event will appear as MET plus a single $b$-jet for which there have so far been only null searches at the LHC [46] with a current bound of $\sim 1 \mathrm{fb}$ on the production cross section (after suitable cuts).
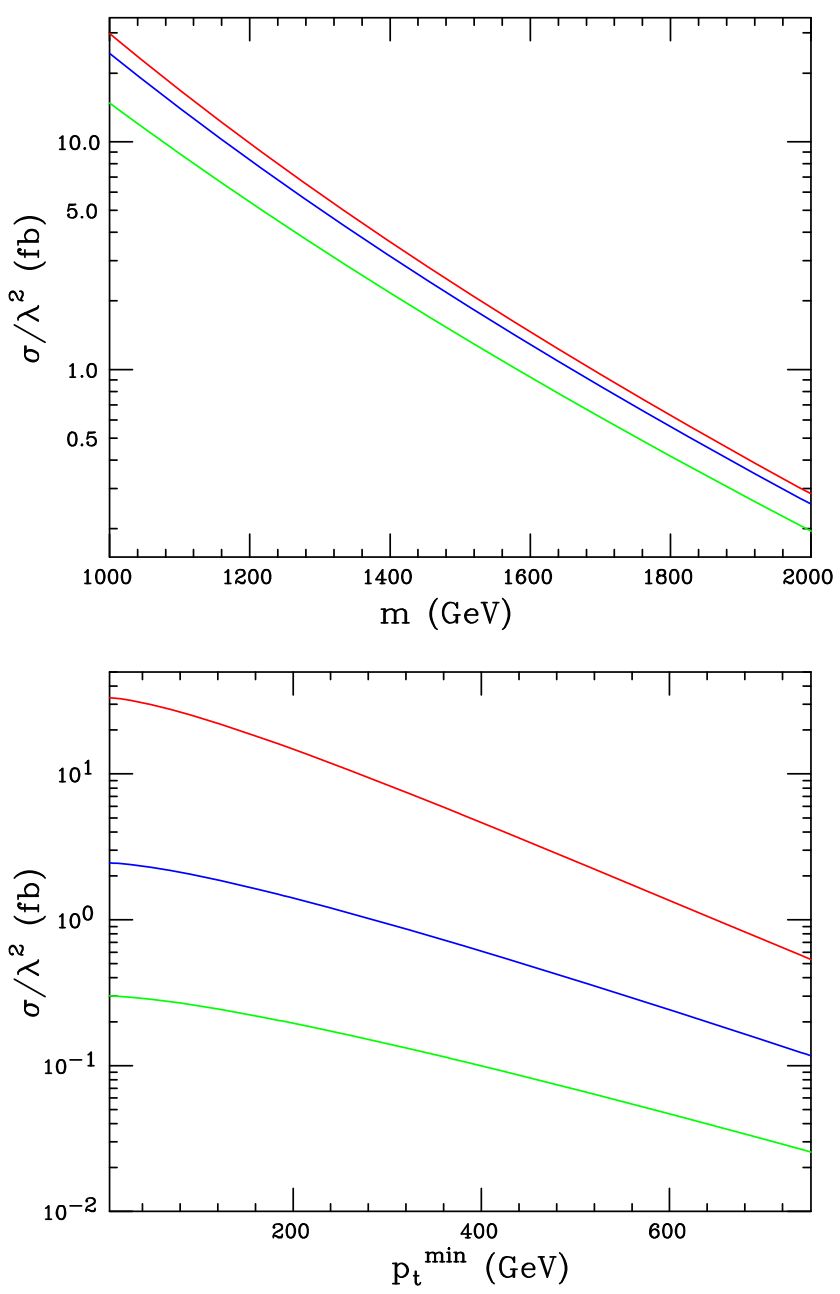

FIG. 8. (Top) lowest order cross section for single $B$ production in association with $S / V$ as a function of the $B$ mass. From top to bottom a cut of $p_{T}(S, V)>50,100,200 \mathrm{GeV}$, respectively, has been applied. (Bottom) Same as in the top panel but now as a function of $p_{T}^{\min }(S, V)$ for, from top to bottom, a $B$ mass of 1, 1.5, or $2 \mathrm{TeV}$, respectively, has been assumed. In both panels a cut of $\left|\eta_{V, S}\right|<2.5$ has also been applied.

Finally we remind the reader that the possibility of the $\bar{B} B \rightarrow \bar{b} b+S S / V V / V S$ final state at the LHC is just one example of what may happen for any of the $\bar{F} F$ portal matter final states discussed above. As mentioned earlier one should also insure that the $\bar{t} t+S, V$ final state is also explored since it is qualitatively similar to the case of $F=B$ except that top reconstruction is somewhat less efficient than is the tagging of high- $p_{T} b$-jets. In the case of $F=T$, the top quark polarization in the $T \rightarrow t V, S$ decays can be used to obtain an additional handle on the chirality structure of the $T t V$ and $T t S$ vertices [47] which can yield important information about the portal matter multiplet structure, in particular, allowing one to distinguish isodoublet from isosinglet scenarios. Also, one should not ignore the pair production of the leptonic PM states at the LHC through their electroweak 


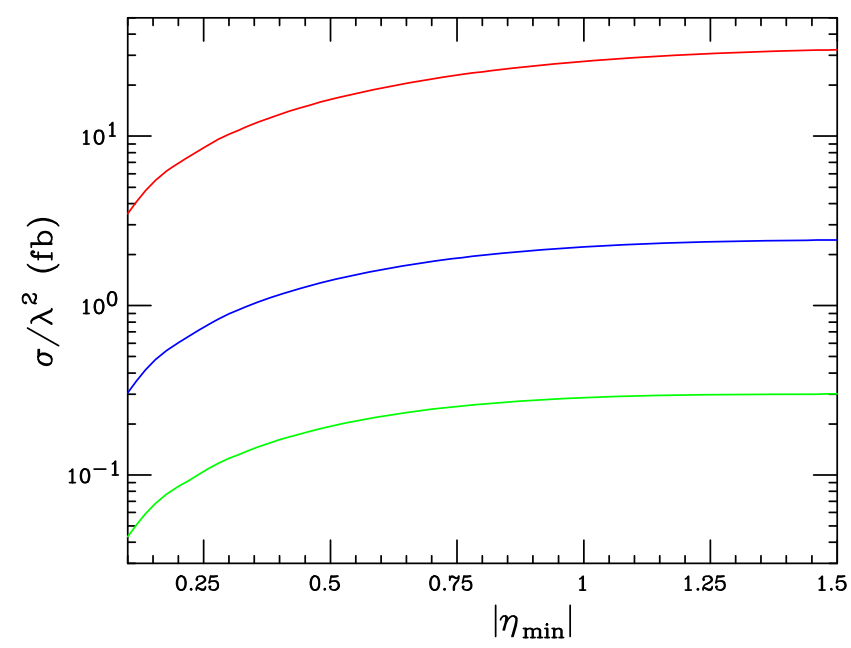

FIG. 9. Associated production cross section for $B+V / S$ as a function of the $\left|\eta_{V, S}\right|_{\min }$; from top to bottom, we assume a $B$ mass of $1,1.5$, or $2 \mathrm{TeV}$, respectively.

interactions which can only have sizable rates at somewhat lower masses and that can lead to MET $+V, S$ when $F=N$ or opposite sign, same flavor leptons $+V, S$ final states when $F=E, M$.

\section{SUMMARY AND CONCLUSIONS}

The generation of the kinetic mixing between the dark photon and the hypercharge gauge boson of the Standard Model is a necessary element in the construction of the dark photon mediator model. To accomplish this KM requires the existence of portal matter simultaneously carrying both dark and SM charges and if this matter is fermionic, as we argue above, it must be vectorlike with respect to the SM to avoid anomalies and the experimental constraints from arising from both precision electroweak data and Higgs decays. Among the set of possible SM transformation properties for these states, one can only chose from among those that allow for the portal matter to decay. This being the case, we demonstrated that the only way to do this at tree level, given that the dark Higgs is a $\mathrm{SM}$ singlet, is to require that the portal matter transform as vectorlike versions of the SM fields themselves. Of these five possible states, those that are also singlets under the $S U(2)_{W}$ gauge group presents us with the simplest possibilities and it is these cases that we have mainly focused on here. However, it is quite likely that in more UV-complete frameworks the portal matter sector may be some more complex and may be a more interesting combination of these different vectorlike states. In particular, we have found that if the portal matter and the dark Higgs, which is responsible for generating the mass of the dark photon, carry the same dark charge, then the vev of the dark Higgs will induce a mixing between the portal matter and the analogous SM field carrying the same SM quantum numbers thus allowing for the desired (prompt) decay path. This same mixing leads to parity-violating dark photon couplings to (only) the specific SM fields which are the conventional analogs of the portal matter. If the portal matter transforms like the $e_{R}$, these parityviolating interactions may be probed by, e.g., the MOLLER experiment. Similarly, if the portal matter transforms as $\mu_{R}$, this same mixing will also lead to potential new contributions to the muon's g- 2 . This mixing is, in particular, also responsible for the specific prompt portal matter decays $F \rightarrow f V, S$, which are the (by far) dominant modes for these new states, that can be observable at the LHC and future colliders. As we have shown, although these portal matter fields are vectorlike fermions similar to the ones most commonly discussed in the literature, they do not decay into the familiar and usually sought $H, W^{ \pm}$or $Z$ final states with any significant branching fractions so that these final states are not the ones to be employed in searching for portal matter. Here we discussed at some length in the analysis above the specific case when the portal matter transforms as a color triplet, i.e., similar to $b_{R}$ in the SM, which leads, in the case of portal matter pair production, to $b$-jet pairs (which can be used to trigger on these events) plus possible MET in a manner similar to (but with larger cross sections than) bottom squarks, or, more interestingly, $b$-jets plus very highly boosted displaced lepton-jet signals at the LHC. In most of the model parameter space these lepton jets are much more highly boosted than what usually results from the signal models that have been previously employed in all the LHC searches and can pose a serious resolution challenge for collider detectors. Qualitatively similar signatures are possible if the portal matter transforms like $t_{R}$ except for the presence of boosted top jets instead of $b$-jets in the final state. Also in the case of a $b_{R}$-like portal matter (but not in the $t_{R}$-like case), the $\mathrm{O}(1)$ couplings to the dark Higgs allow for the possibility that single production of portal matter may occur at significant rates with a unique signature having only a single triggering jet.

The exploration of the physics of portal matter as discussed above, especially at colliders, is just at its beginning stages and there are multiple directions which can be pursued which we leave for future study.

\section{ACKNOWLEDGMENTS}

The author would like to particularly thank J. L. Hewett and T.D. Rueter for discussions. He would also like to especially thank the members of the SLAC ATLAS Collaboration, in particular, Su Dong, C. Young, M. Diamond, R. Bartoldus, M. Kagan, C. Vernieri and A. Schwartzman, for very valuable discussions on the long-lived particles and lepton-jets analyses with ATLAS. This work was supported by the Department of Energy, Contract No. DE-AC02-76SF00515. 


\section{APPENDIX: COUPLING AND INTERACTION SUMMARY}

We collect and review in this appendix some useful approximate expressions for the couplings of the various physical fields that we have employed in the present analysis.

Recall that we assume that the dark photon has a mass in (roughly) the range $\sim 0.1-1 \mathrm{GeV}$ implying that the singlet dark Higgs vev is $v_{s} \lesssim \mathrm{a}$ few GeV; the portal matter-SM dark Higgs couplings, $\lambda_{i}$, are assumed to be $\mathrm{O}(1)$. Correspondingly, a pair of mass parameters, $X_{i}=$ $\lambda_{i} v_{s} / \sqrt{2} \sim \mathrm{GeV}$ can also be defined for convenience. Given the experimental constraints and future experimental interest, we have consequently focused on KM parameter values of $\epsilon \sim 10^{-(3-5)}$. Since portal matter fields are generally much heavier than their corresponding SM partners, of order several hundred $\mathrm{GeV}$ to more than $1 \mathrm{TeV}$, to leading order and for the isosinglet models we consider, we can treat the left-handed fermion mixing matrix as approximately diagonal, i.e., $U_{L} \simeq I$ and deal only with a nontrivial $U_{R}$ in our phenomenological analyses. Since $U_{L} \simeq I$, one finds that the decays of portal matter fields into their SM partners and the SM fields $W^{ \pm}$, $Z, H$ are relatively suppressed. As is usual, all the SM fields have an interaction with the dark photon given by ee $Q_{e m}$. However, the nontrivial $U_{R}$ mixing induced by the dark Higgs does three things: (i) it leads to an additional contribution, corresponding to a parity-violating, righthanded coupling, of the dark photon to the SM field, $g_{D}\left(C_{R}\right)_{11} \simeq g_{D}\left(X_{1} / m_{1}\right)^{2}$, but only for the SM field which has this specific portal matter partner (here of mass $m_{1}$ ). A nontrivial $\left(C_{R}\right)_{11}$ leads to all of the leptonic portal matter discussion above involving the dark photon. We note that $g_{D}\left(C_{R}\right)_{11}$ is expected to be not very different than $e \epsilon$ in magnitude and so it is useful to define a relative strength parameter $y=g_{d}\left(C_{R}\right)_{11} / 2 e \epsilon$. (ii) This same nontrivial mixing matrix also leads to an off-diagonal coupling of the dark photon between this portal field and its SM partner, e.g., $g_{D}\left(C_{R}\right)_{12} \simeq g_{D} X_{1} / m_{1}$, allowing for a new contribution to $\mathrm{g}-2$, for the portal matter to decay and providing, e.g., a production signature at the LHC as discussed above. Finally, (iii) since this portal matter-SM mixing is induced by an off-diagonal coupling of the dark Higgs, this off-diagonal interaction remains in the mass eigenstate basis, e.g., $\beta_{21} \simeq \lambda_{1} X_{1} /\left(\sqrt{2} m_{1}\right)$, which allows for the dark Higgs couplings contributing to $g-2$ and for a second decay channel of the portal matter fields at the LHC.
[1] M. Kawasaki and K. Nakayama, Annu. Rev. Nucl. Part. Sci. 63, 69 (2013).

[2] P. W. Graham, I. G. Irastorza, S. K. Lamoreaux, A. Lindner, and K. A. van Bibber, Annu. Rev. Nucl. Part. Sci. 65, 485 (2015).

[3] For a recent review of WIMPs, see G. Arcadi, M. Dutra, P. Ghosh, M. Lindner, Y. Mambrini, M. Pierre, S. Profumo, and F. S. Queiroz, Eur. Phys. J. C 78, 203 (2018).

[4] J. Alexander et al., arXiv:1608.08632.

[5] M. Battaglieri et al., arXiv:1707.04591.

[6] There has been a huge amount of work on this subject; see, for example, D. Feldman, B. Kors, and P. Nath, Phys. Rev. D 75, 023503 (2007); D. Feldman, Z. Liu, and P. Nath, Phys. Rev. D 75, 115001 (2007); M. Pospelov, A. Ritz, and M. B. Voloshin, Phys. Lett. B 662, 53 (2008); M. Pospelov, Phys. Rev. D 80, 095002 (2009); H. Davoudiasl, H. S. Lee, and W. J. Marciano, Phys. Rev. Lett. 109, 031802 (2012); Phys. Rev. D 85, 115019 (2012); R. Essig et al., arXiv:1311.0029; E. Izaguirre, G. Krnjaic, P. Schuster, and N. Toro, Phys. Rev. Lett. 115, 251301 (2015); M. Khlopov, Int. J. Mod. Phys. A 28, 1330042 (2013); for a general overview and introduction to this framework, see D. Curtin, R. Essig, S. Gori, and J. Shelton, J. High Energy Phys. 02 (2015) 157.

[7] B. Holdom, Phys. Lett. 166B, 196 (1986); Phys. Lett. B 178, 65 (1986); K. R. Dienes, C. F. Kolda, and J. MarchRussell, Nucl. Phys. B492, 104 (1997); F. Del Aguila, Acta
Phys. Pol. B 25, 1317 (1994); K. S. Babu, C. F. Kolda, and J. March-Russell, Phys. Rev. D 54, 4635 (1996); T. G. Rizzo, Phys. Rev. D 59, 015020 (1998).

[8] See, however, some earlier general discussions in B. Patt and F. Wilczek, arXiv:hep-ph/0605188; H. Davoudiasl, R. Kitano, T. Li, and H. Murayama, Phys. Lett. B 609, 117 (2005); D. McKeen, M. Pospelov, and A. Ritz, Phys. Rev. D 86, 113004 (2012); B. Batell, M. Pospelov, A. Ritz, and Y. Shang, Phys. Rev. D 81, 075004 (2010).

[9] For a recent overview of vectorlike quarks and original references, see C. Y. Chen, S. Dawson, and E. Furlan, Phys. Rev. D 96, 015006 (2017); for a corresponding recent overview of vectorlike leptons and original references, see Z. Poh and S. Raby, Phys. Rev. D 96, 015032 (2017); for a general recent review of VLF, see V. Peralta, arXiv:1712 .06193 .

[10] M. Aaboud et al. (ATLAS Collaboration), Phys. Rev. Lett. 121, 211801 (2018); A. M. Sirunyan et al. (CMS Collaboration), J. High Energy Phys. 08 (2018) 177; CMS Collaboration, CERN Report No. CMS-PAS-EXO18-005, 2018.

[11] See the first two papers in Ref. [7].

[12] T. G. Rizzo (in preparation).

[13] N. Baltzell et al. (HPS Collaboration), Nucl. Instrum. Methods Phys. Res., Sect. A 859, 69 (2017); M. De Napoli (HPS Collaboration), EPJ Web Conf. 142, 01011 (2017); 
P. H. Adrian et al. (HPS Collaboration), Phys. Rev. D 98, 091101 (2018).

[14] For more information about the LDMX experiment, see https://confluence.slac.stanford.edu/display/MME/Light+Dark +Matter+Experiment; T. Raubenheimer et al., arXiv:1801 .07867; and also T. Akesson et al. (LDMX Collaboration), arXiv:1808.05219.

[15] T. G. Rizzo, J. High Energy Phys. 07 (2018) 118.

[16] T. G. Rizzo, J. High Energy Phys. 10 (2018) 069.

[17] The best LHC limits to date are from G. Aad et al. (ATLAS Collaboration), J. High Energy Phys. 11 (2015) 206; and V. Khachatryan et al. (CMS Collaboration), J. High Energy Phys. 02 (2017) 135.

[18] This possibility was first discussed in another context in $\mathrm{H}$. Davoudiasl, H. S. Lee, and W. J. Marciano, Phys. Rev. D 86, 095009 (2012).

[19] C. D. Carone, S. Chaurasia, and T. V. B. Claringbold, Phys. Rev. D 99, 015009 (2019).

[20] Even lighter leptonic VLF states may be possible; see, for example, D. Egana-Ugrinovic, M. Low, and J. T. Ruderman, J. High Energy Phys. 05 (2018) 012.

[21] This possibility was first discussed in the work of $\mathrm{H}$. Davoudiasl, H.-S. Lee, and W. J. Marciano in Ref. [6] and also in H. Davoudiasl, H. S. Lee, and W. J. Marciano, Phys. Rev. D 89, 095006 (2014); see also M. Pospelov, Phys. Rev. D 80, 095002 (2009).

[22] M. S. Chanowitz and M. K. Gaillard, Nucl. Phys. B261, 379 (1985); B. W. Lee, C. Quigg, and H. B. Thacker, Phys. Rev. D 16, 1519 (1977); J. M. Cornwall, D. N. Levin, and G. Tiktopoulos, Phys. Rev. D 10, 1145 (1974); 11, 972(E) (1975); G. J. Gounaris, R. Kogerler, and H. Neufeld, Phys. Rev. D 34, 3257 (1986).

[23] For a recent review, see B. M. Roberts, V. A. Dzuba, and V. V. Flambaum, Annu. Rev. Nucl. Part. Sci. 65, 63 (2015).

[24] J. Benesch et al. (MOLLER Collaboration), arXiv:1411 .4088; D. Androic et al. (Qweak Collaboration), Nature (London) 557, 207 (2018); K. Aulenbacher and A. Jankowiak, Polarized Electrons and Positrons at the Mesa Accelerator (World Scientific, Singapore, 2011).

[25] Jefferson Lab Qweak Collaboration, Nature (London) 557, 207 (2018).

[26] M. Tanabashi et al. (Particle Data Group), Phys. Rev. D 98, 030001 (2018).

[27] P. L. Anthony et al. (SLAC E158 Collaboration), Phys. Rev. Lett. 95, 081601 (2005).

[28] For a recent review of the status of the muon's g-2, see F. Jegerlehner, Acta Phys. Pol. B 49, 1157 (2018); T. Ishikawa, N. Nakazawa, and Y. Yasui, Phys. Rev. D 99, 073004 (2019).

[29] J. P. Leveille, Nucl. Phys. B137, 63 (1978).

[30] C. Y. Chen, H. Davoudiasl, W. J. Marciano, and C. Zhang, Phys. Rev. D 93, 035006 (2016); J. L. Feng, B. Fornal, I. Galon, S. Gardner, J. Smolinsky, T. M. P. Tait, and P. Tanedo, Phys. Rev. D 95, 035017 (2017).
[31] For some constraints on such a scenario, see S. Bilmis, I. Turan, T. M. Aliev, M. Deniz, L. Singh, and H. T. Wong, Phys. Rev. D 92, 033009 (2015).

[32] See, for example, A. N. Khan and D. W. McKay, J. High Energy Phys. 07 (2017) 143.

[33] We employ numerical estimates based on M. Czakon and A. Mitov, Comput. Phys. Commun. 185, 2930 (2014).

[34] M. Bauer, P. Foldenauer, and J. Jaeckel, J. High Energy Phys. 07 (2018) 094; P. Ilten, Y. Soreq, M. Williams, and W. Xue, J. High Energy Phys. 06 (2018) 004.

[35] T. G. Rizzo, Phys. Rev. D 22, 722 (1980); W. Y. Keung and W. J. Marciano, Phys. Rev. D 30, 248 (1984).

[36] See, for example, O. Ruchayskiy and A. Ivashko, J. Cosmol. Astropart. Phys. 10 (2012) 014.

[37] M. Aaboud et al. (ATLAS Collaboration), J. High Energy Phys. 11 (2017) 195; A. M. Sirunyan et al. (CMS Collaboration), Phys. Lett. B 778, 263 (2018).

[38] LHC SUSY Cross Section Working Group, https://twiki .cern.ch/twiki/bin/view/LHCPhysics/SUSYCrossSections.

[39] W. Beenakker, C. Borschensky, M. Kramer, A. Kulesza, and E. Laenen, J. High Energy Phys. 12 (2016) 133; M. B. Wise and Y. Zhang, J. High Energy Phys. 06 (2018) 053.

[40] C. Alpigiani et al., A letter of intent for MATHUSLA: A dedicated displaced vertex detector above ATLAS or CMS, CERN Reports No. CERN-LHCC-2018-025, No. LHCC-I031, 2018.

[41] A. Ariga et al. (FASER Collaboration), arXiv:1811.10243.

[42] N. Arkani-Hamed and N. Weiner, J. High Energy Phys. 12 (2008) 104; M. Baumgart, C. Cheung, J. T. Ruderman, L. T. Wang, and I. Yavin, J. High Energy Phys. 04 (2009) 014; A. Falkowski, J. T. Ruderman, T. Volansky, and J. Zupan, Phys. Rev. Lett. 105, 241801 (2010); J. High Energy Phys. 05 (2010) 077; C. Cheung, J. T. Ruderman, L. T. Wang, and I. Yavin, J. High Energy Phys. 04 (2010) 116; G. Barello, S. Chang, C. A. Newby, and B. Ostdiek, Phys. Rev. D 95, 055007 (2017).

[43] For some related LHC searches, see M. Aaboud et al. (ATLAS Collaboration), Phys. Rev. D 99, 052005 (2019); A. M. Sirunyan et al. (CMS Collaboration), J. High Energy Phys. 02 (2019) 179; Phys. Rev. D 99, 032011 (2019).

[44] G. Aad et al. (ATLAS Collaboration), J. High Energy Phys. 11 (2014) 088; 02 (2016) 062; M. Del Gaudio (ATLAS Collaboration), Proc. Sci., EPS-HEP2017 (2018) 690; ATLAS note ATLAS-CONF-2016-042; V. Khachatryan et al. (CMS Collaboration), Phys. Lett. B 752, 146 (2016); CMS Collaboration, CERN Report No. CMSPAS-HIG-18-003, 2018.

[45] J. de Favereau, C. Delaere, P. Demin, A. Giammanco, V. Lemaître, A. Mertens, and M. Selvaggi (DELPHES 3 Collaboration), J. High Energy Phys. 02 (2014) 057.

[46] M. Aaboud et al. (ATLAS Collaboration), Eur. Phys. J. C 78, 18 (2018); CMS Collaboration, CERN Report No. CMS-PAS-B2G-15-007.

[47] See, for example, H. Zhou and N. Liu, Phys. Lett. B 791, 1 (2019). 